\title{
Individual and partnership characteristics associated with consistent condom use in a cohort of cisgender men who have sex with men and transgender women in Nigeria
}

Oluwasolape Olawore ${ }^{1 *}$ (D), Trevor A. Crowell ${ }^{2,3}$, Sosthenes C. Ketende ${ }^{1}$, Habib O. Ramadhani ${ }^{4}$, Hongjie Liu ${ }^{5}$, Julie A. Ake ${ }^{2}$, Afoke Kokogho6,7, Sylvia Adebajo ${ }^{8}$, Man E. Charurat ${ }^{4}$, Rebecca G. Nowak ${ }^{4}$ Stefan D. Baral ${ }^{1}$ and on behalf of the TRUST/RV368 Study Group

\begin{abstract}
Background: This study reports on the individual and partnership characteristics that influence consistent condom use in cisgender men who have sex with men (MSM) and transgender women (TGW) attending trusted community centers that provide HIV prevention and treatment services in Nigeria.

Methods: Adults assigned male at birth who reported anal sex with male partners who enrolled between March 2013-2019 and had information about at least one male sexual partner were included in these analyses. At enrollment and follow-up visits every 3 months for up to 18 months, participants were administered detailed questionnaires that collected information about demographics, sexual practices, HIV risk behaviors, and characteristics and behaviors of their partners in the previous year (at enrollment) or the preceding 3 to 6 -months (at follow-up visits). Logistic regression models with generalized estimating equations were used to assess the odds ratio (OR) and 95\% confidence intervals (Cl) of individual, partner, and partnership characteristics associated with consistent condom use (CCU). A participant was defined as consistently using condom if they reported always using condoms all the time they had insertive, receptive or both types of anal sex with a male partner.
\end{abstract}

Results: At the individual level, CCU was positively associated with higher education, disclosure of key population status to a healthcare worker and negatively associated with poor access to condoms. At the partner and partnership level, CCU was associated with partners with higher education (aOR: 1.36; 95\% Cl: 1.07-1.72), casual relationships (aOR: 1.22; 95\% Cl: 1.11-1.34) and relationships in which partners encouraged the participant to use condoms with other partners (aOR: 1.14; 95\% Cl: 1.02-1.28). Relationships in which the partner was married to a woman and/or the partner's HIV status positive or unknown were negatively associated with CCU.

\footnotetext{
* Correspondence: oolawor1@jhmi.edu

'Johns Hopkins Bloomberg School of Public Health, 615 North Wolfe Street Suite 3507, Baltimore, MD 21205, USA

Full list of author information is available at the end of the article
}

C C The Author(s). 2021 Open Access This article is licensed under a Creative Commons Attribution 4.0 International License, which permits use, sharing, adaptation, distribution and reproduction in any medium or format, as long as you give appropriate credit to the original author(s) and the source, provide a link to the Creative Commons licence, and indicate if changes were made. The images or other third party material in this article are included in the article's Creative Commons licence, unless indicated otherwise in a credit line to the material. If material is not included in the article's Creative Commons licence and your intended use is not permitted by statutory regulation or exceeds the permitted use, you will need to obtain permission directly from the copyright holder. To view a copy of this licence, visit http://creativecommons.org/licenses/by/4.0/. The Creative Commons Public Domain Dedication waiver (http://creativecommons.org/publicdomain/zero/1.0/) applies to the data made available in this article, unless otherwise stated in a credit line to the data. 
Conclusions: These findings suggest that individuals in relationships where partners were more open and encouraged safer sex were more likely to consistently use condoms. HIV prevention programs should consider leveraging communication to sexual partners to encourage condom use as this may support condom use with other sexual partners. Given sustained and growing HIV and STI epidemics among MSM and TGW, even with preexposure prophylaxis scale-up, it is crucial to continue to study optimal implementation strategies to increase condom use.

Keywords: Men who have sex with men, Nigeria, condoms, Networks, HIV, Epidemiology, Sexual and gender minorities

\section{Background}

Even in the context of a mixed HIV epidemic in Nigeria, cisgender men who have sex with men (MSM) and transgender women (TGW) bear a disproportionate burden of infection with estimated HIV prevalence $18-66 \%$ compared to about $4 \%$ among reproductive aged adults [1, 21, 42, 43]. This increased burden is due to the high HIV transmission risks associated with condomless anal intercourse with viremic partners [3, 13, 29]. It has been estimated that the 5-year cumulative incidence of HIV among MSM would be reduced by $80-98 \%$ if the efficiency of HIV transmission in anal sex was similar to the efficiency of transmission in condomless vaginal sex [3]. While the use of pre-exposure prophylaxis (PrEP) is effective in reducing transmission of HIV, it is not routinely available in many sub-Saharan African (SSA) countries. Thus, consistent condom use (CCU) remains a cost-effective HIV prevention strategy writ large.

Individual-level determinants of CCU among MSM in Nigeria have been shown to include knowledge of HIV transmission, frequency of sex, and alcohol or drug use before or during sex [39]. However, factors influencing condom use may not be fully captured by a single measure and understanding the dynamics between the attributes of the sexual relationship, partner characteristics, and individual level factors is necessary to fully characterize the determinants of condom usage and opportunities for intervention [5, 24, 41].

Among MSM, research has consistently shown unprotected anal intercourse is more likely in committed or more serious relationships compared to relationships that are more casual $[7,15,27,40]$. This dynamic has been attributed to higher levels of trust and familiarity with committed partners and the perception that condom use may affect intimacy $[10,15,18,23,48]$. Perceived and actual partner characteristics have also been shown to influence individual decisions to use condom. For example, MSM at risk for HIV may be more likely to use condom with partners they perceive to be more likely to be living with HIV and serosorting is common among MSM when both partners have disclosed their
HIV statuses [27, 35, 47]. Other factors such as age difference between partners, sexual partner concurrency, and partner norms around condoms use may also impact $\mathrm{CCU}[5,26,27]$.

Although TGW have been shown to have higher odds of reporting condomless receptive anal sex with male partners compared to MSM, few studies have characterized the factors that drive this association in TGW specifically [32]. A study among TGW in China found that subjective norms, condom use attitude, condom use skills, and self-efficacy were associated with frequent condom use and knowledge of HIV was not related to condom use $[6,45]$. Other factors that have been shown to be associated with condomless sex among TGW include feminizing medical intervention, factors related to sex-work [6].

While various studies have provided evidence for the partner and relationship characteristics that may influence individual decisions to consistently use condoms, there is limited research on the subject matter among MSM and TGW in sub-Saharan Africa, where cultural factors, resource limitations and other factors may influence the dynamics of condom negotiation and decisionmaking in ways that differ from those in westernized settings. Given limited condom uptake among cisgender MSM and TGW in Nigeria $[8,39,44]$ and the continued importance of characterizing optimal strategies to encourage CCU, we assessed the individual, relationship and partner-level factors associated with CCU among a cohort of cisgender MSM and TGW enrolled in two community-engaged clinics in Abuja and Lagos, Nigeria, thus identifying characteristics that may aid in developing targeted strategies.

\section{Methods \\ Study design and population}

TRUST/RV368 study is a prospective cohort study in Lagos and Abuja, Nigeria that assesses the effectiveness of network-based recruitment of MSM and TGW into an HIV prevention and treatment program. As described elsewhere, participants are recruited into the study using respondent-driven sampling; RDS [2, 8, 33]. Briefly, participants are eligible to participate if they were assigned 
male at birth, are aged $\geq 16$ years (Abuja) or $\geq 18$ years (Lagos), and reported receptive or insertive anal intercourse with a male partner in the past year. Initial seed participants were recruited through community-based sampling of individuals who were identified to be wellconnected within the population of interest. Each seed was given three RDS coupons to distribute to eligible peers and each individual subsequently recruited was also given three coupons to distribute until equilibrium was achieved.

At enrollment and follow-up visits every 3 months for up to 18 months, participants were administered a detailed questionnaire that collected information about demographics, sexual practices, and HIV risk behaviors in the previous year (at enrollment) or the preceding 3 to 6-months (at follow-up visits). Participants also provided information related to demographics and risk behaviors of up to five of their sexual partners from the past year. At each visit, participants were counselled and screened for HIV and other sexually transmitted infections (STIs). Blood samples were tested for HIV using a parallel testing algorithm of two rapid tests according to national guidelines in Nigeria [28]. The questionnaires used (supplemental file) were developed for the study incorporating modifications of previously validated scales such as the PHQ9 depression module and the World Bank's Social Capital Tool [22, 46].

Baseline data for participants enrolled in the study between April 2013 and April 2019 who provided information on at least one sexual partner at enrollment were included in these analyses. All participants provided written informed consent in English or Hausa. The study was approved by the University of Maryland Baltimore Institutional Review Board (IRB); the Federal Capital Territory Health Research Ethics Committee, Abuja; and Walter Reed Army Institute of Research IRB.

\section{Outcomes of interest}

The three outcomes of interest were CCU with insertive anal sex, CCU with receptive anal sex, and CCU with both types of anal sex in the past year. For each anal sex type, participants were asked "In the past year, when you had anal sex with [partner name], how often was a condom used?". A participant was defined as consistently using condom if they reported always using condoms all the time they had insertive, receptive or both types of anal sex with a male partner. Each outcome was examined in a separate model.

\section{Predictors of interest}

\section{Participant-level factors}

Participant-level factors of interest included demographic characteristics such as age, education status, marital status, sexual orientation and gender identity.
Other participant-level variables of interest were disclosure of sexual or gender identity to a healthcare worker, drinking habits in the past 30 days, access to condoms, knowledge of HIV risks, history of STI and HIV testing, previous STI diagnosis, self-reported HIV status, and HIV testing results at baseline.

\section{Partner and relationship-level factors}

Partner and relationship-level factors were all reported from the perspective of the participant. Partner-level variables included partner's age, education status, sexual orientation, marital status, sexual concurrency, condom use with their other partners, alcohol or drug use, STI history, and HIV status.

Relationship-level variables included relationship type, age difference between participant and partner socioeconomic difference between participant and partner, frequency of sexual intercourse with partner, how often HIV was discussed in relationship, and HIV serostatus concordance based on self-reported HIV status. Sexual network density-based on the answer to the question "Does sexual partner one know sexual partner two?" and so on up to the fifth sexual partner as necessary-was also assessed. Sexual network density was defined as degree of connection between the sexual partners of a participant and was calculated as the total number of actual ties divided by the total number of potential ties. Network density scores were dichotomized with $<50 \%$ considered "smaller" and $\geq 50 \%$ considered "larger" network density.

\section{Statistical analyses}

Demographic characteristics and sexual behaviors of study participants and at most five of their partners were examined at enrollment using descriptive statistics.

Odds ratios (ORs) and adjusted odds ratios (aORs) with corresponding 95\% confidence intervals (CI) of participant and partnership covariates associated with $\mathrm{CCU}$ during insertive, receptive, and both types of anal sex were calculated using logistic regression with generalized estimating equations to account for clustering within participants. Variables significant at $p \leq 0.10$ and prespecified variables including age, marital status, gender identity, and education status previously known to be associated with CCU were included in the multiple regression analyses regardless of statistical significance. 10.05 was considered significant in the multiple regression analyses. Analyses were carried out as complete case analyses and refusal/don't know responses were kept as categories the final model where applicable. Sub-group analyses assessing the associated factors in the two KP groups separately i.e., MSM and TGW were also carried. All analyses were performed using Stata (version 15; StataCorp LP, College Station, Texas USA). 
Table 1 Characteristics of men who have sex with men and transgender women at enrollment into the TRUST/RV368 cohort in Abuja and Lagos, Nigeria

\begin{tabular}{ll}
\hline Participant Characteristics $(\boldsymbol{N}=\mathbf{1 7 8 6})$ & $\mathbf{n}(\%)$ \\
\hline Site & \\
$\quad$ Abuja & $1324(74.1)$ \\
Lagos & $462(25.9)$
\end{tabular}

\section{Age categories}

$$
\begin{aligned}
& \text { 16-19 years } \\
& \text { 20-24 years } \\
& \text { 25-29 years } \\
& \text { 30-34 years } \\
& 35 \text { and older } \\
& \text { Missing }
\end{aligned}
$$

\section{Sexual orientation}

Gay or homosexual

Bisexual

Other

Refusal/don't know /missing

\section{Gender identity}

$$
\begin{aligned}
& \text { Male } \\
& \text { Female } \\
& \text { Both/Other } \\
& \text { Refusal/don't know/missing } \\
& \text { Education level } \\
& \text { None/Primary education only } \\
& \text { Junior secondary only } \\
& \text { Senior secondary } \\
& \text { Higher than senior secondary } \\
& \text { Refusal/don't know/missing }
\end{aligned}
$$

\section{Education level}

\section{Marital status}

Single/never married

Married/cohabitating with a woman

Cohabitating with a man

Divorced/separated/widowed/other

Refusal/don't know/missing

\section{Ever disclosed MSM status to healthcare worker}

No

Yes

Refusal/don't know

Missing

\section{Access to condoms}

Gets condoms for free

Buys condoms

Gets for free and buys them

Neither buys condom nor gets them for free

$96(5.4)$

$920(51.5)$

$661(37.0)$

$22(1.2)$

$1612(90.3)$

$92(5.2)$

$23(1.3)$

$44(2.5)$

$15(0.84)$

$1204(67.4)$

$565(31.6)$

$5(0.3)$

$12(0.7)$

$531(29.7)$

$683(38.2)$

$502(28.1)$
Table 1 Characteristics of men who have sex with men and transgender women at enrollment into the TRUST/RV368 cohort in Abuja and Lagos, Nigeria (Continued)

\begin{tabular}{ll}
\hline Participant Characteristics $(\boldsymbol{N}=\mathbf{1 7 8 6})$ & $\mathbf{n}(\%)$ \\
\hline Refusal/don't know/missing & $27(1.5)$ \\
Episodes of heavy drinking in the past 30 days & $143(8.0)$ \\
Social Cohesion & \\
$\quad$ Low social cohesion & $911(51.0)$ \\
Medium social cohesion & $347(19.4)$ \\
High social cohesion & $528(29.6)$
\end{tabular}

\section{Knowledge of HIV risks}

a.) What type of sex is associated with highest HIV transmission risk?

$\begin{array}{ll}\text { All types of sex carry equal risk } & 1027(57.5) \\ \text { Anal sex } & 384(21.5) \\ \text { Vaginal sex } & 237(13.3) \\ \text { Oral } & 60(3.4) \\ \text { Refusal/Don't know } & 62(3.5) \\ \text { Missing } & 16(0.9)\end{array}$

b.) What type of anal sex is associated with the highest HIV transmission risk?

$\begin{array}{ll}\text { All types of anal sex carry equal risk } & 784(43.9) \\ \text { Receptive anal sex } & 735(41.2) \\ \text { Insertive anal sex } & 169(9.5) \\ \text { Refusal/don't know } & 78(4.4) \\ \text { Missing } & 20(1.1)\end{array}$

\section{Any STI test in the past 12 months}

No

$1179(66.0)$

Yes

$592(33.2)$

Refusal/don't know $3(0.2)$

Missing

$12(0.7)$

Any STI diagnosis in the past 12 months

No

$1358(76.0)$

Yes

$388(21.7)$

Refusal/don't know

$9(0.5)$

Missing

$31(1.7)$

How worried have you been about HIV in the past 12 months?

Not at all worried

$846(47.4)$

Somewhat/ a little worried

$468(26.2)$

Very worried

$452(25.3)$

Refusal/don't know/missing

$20(1.1)$

Ever previously tested for HIV?

No

$358(20.0)$

Yes, once

$422(23.6)$

Yes, more than once

989 (55.4)

Refusal/don't know/missing

$17(1.0)$ 
Table 1 Characteristics of men who have sex with men and transgender women at enrollment into the TRUST/RV368 cohort in Abuja and Lagos, Nigeria (Continued)

\begin{tabular}{ll}
\hline Participant Characteristics $(\boldsymbol{N}=\mathbf{1 7 8 6})$ & $\mathbf{n}(\%)$ \\
\hline Self-reported HIV status & $883(49.4)$ \\
Negative & $487(27.3)$ \\
Positive & $416(23.3)$ \\
Unknown & \\
HIV rapid test results & $936(52.4)$ \\
Negative & $842(47.1)$ \\
Positive & $8(0.5)$ \\
Missing & \\
Sexual network density & $561(31.4)$ \\
Smaller network density & $956(53.5)$ \\
Larger network density & $269(15.1)$ \\
Unknown network density &
\end{tabular}

\section{Results}

Between April 2013 and April 2019, 2591 eligible MSM and TGW participants were recruited into the study. Of those, 1786 reported on a total of 6432 sexual partners at enrollment and were included in these analyses. The average sex network size was 3.6.

\section{Participant characteristics}

An overview of the demographic and behavioral characteristics of the participants in this study is presented in Table 1 . Seventy-four percent $(n=1324)$ of the participants were recruited from Abuja and the median age of the participants was 24 years (interquartile range [IQR]: 21-27). Eighty percent $(n=1424)$ self-identified as male and the median number of sexual partners reported in the past year was 4 (IQR: 2-8). Five hundred and thirty (30\%) participants reported being gay or homosexual while $70 \%$ reported being bisexual $(n=1238)$. Most of the participants had a secondary education or higher and majority of them reported being single or never married.

Ninety-six percent $(n=1715)$ of participants reported either buying condoms or getting them for free. A majority of the participants reported that all types of sex were associated with equal risk of HIV transmission, while $22 \%(n=384)$ reported anal sex being the type of sex associated with the highest risk of transmission. Seventy-nine percent $(n=1411)$ of the participants had ever been previously tested for HIV and a quarter of the participants reported being very worried about HIV in the past 12 months Of the 1786 participants included in these analyses, $47 \%$ $(n=842)$ were living with HIV.

\section{Partner and relationship characteristics}

The participant reported characteristics of the partners and relationships are presented in Table 2. The median age of partners reported on was 27 (IQR: 23$32)$ and $65 \%(n=4106)$ of the partners were reported to be casual partners. Most of the partners $(n=5285$; $82 \%)$ were single or never married and 53\% $(n=3418)$ were reported to be bisexual. A majority of the partners had a senior secondary education or higher and more than half of the partners reported on had a socio-economic status that was higher than the participants'.

Sixty-eight percent of the partners reported having multiple regular sexual partners at the same time, while only $15 \%$ of the partners were believed to always consistently use condoms when they had had sex with their other sexual partners. Condom use was encouraged by $52 \%$ of the partners and $18 \%$ of the partners discussed HIV with the participant at least once a week.

The HIV status of $54 \%$ of the partners were unknown by the participants while $6 \%$ of the partners were reported to be HIV positive. Using the self-reported HIV status of the participants and the participant reported HIV status of the partners, $4 \%$ of the partnerships were HIV positive seroconcordant and $75 \%$ of the partnership were HIV-serodiscordant or had unknown seroconcordance status.

\section{Individual characteristics associated with consistent condom use}

The bivariate and multivariable analyses of the participant characteristics associated with CCU with insertive, receptive, both types of anal sex are presented in Table 3 . The results of the analysis of CCU with both types of anal sex are reported here.

In the bivariate analysis, higher education status disclosure of MSM status to a healthcare worker, previous STI and HIV tests, self-reported positive HIV status, and knowledge that anal sex had the highest HIV transmission risk were all positively associated with CCU.

Age between 20 and 24, participants who identified as women, buying condoms or neither buying nor getting condoms for free, being very worried about HIV in the previous 12 months, and a self -reported positive HIV status were all negatively associated with CCU.

In the multivariable analysis (Table 3), higher education status (aOR: 1.80; 95\% CI: 1.10-2.94) and disclosure of MSM status to a healthcare worker (aOR: 1.22 1.001.48) remained positively associated with CCU. All age categories except 35 years or older were negatively associated with CCU and so was neither buying nor getting condoms for free (aOR: 0.26; 95\% CI: 0.11-0.59), and being very worried about HIV in the previous 12 months (aOR: 0.66; 95\% CI: 0.53-0.82). 
Table 2 Partner and partnership characteristics by CCU with both types of anal sex

\begin{tabular}{|c|c|c|c|c|}
\hline Partner and relationship characteristics & $\begin{array}{l}\text { Total } \\
(N=6432)\end{array}$ & $\begin{array}{l}\text { CCU with both types of } \\
\text { anal sex } \\
(N=2853)(44.3 \%)\end{array}$ & $\begin{array}{l}\text { Non-CCU with both types } \\
\text { of anal sex } \\
(N=3579)(55.7 \%)\end{array}$ & $p$ value \\
\hline \multicolumn{5}{|l|}{ Partner age categories } \\
\hline Younger than 25 & $2225(34.6)$ & $1047(36.7)$ & $1178(32.9)$ & \\
\hline $25-34$ & $2904(45.2)$ & $1324(46.4)$ & $1580(44.2)$ & \\
\hline $35-44$ & $546(8.5)$ & $209(7.3)$ & $337(9.4)$ & $<0.001$ \\
\hline 45 and older & $134(2.1)$ & $47(1.7)$ & $87(2.4)$ & \\
\hline Refusal/Don't know & $623(9.7)$ & $226(7.9)$ & $397(11.1)$ & \\
\hline \multicolumn{5}{|l|}{ Partner's education status } \\
\hline None/Primary education only & $168(2.6)$ & $50(1.8)$ & $118(3.3)$ & \\
\hline Junior secondary only & $127(2.0)$ & $49(1.7)$ & $78(2.2)$ & $<0.001$ \\
\hline Senior secondary & 2107 (32.8) & $943(33.1)$ & $1164(32.5)$ & \\
\hline Higher than senior secondary & $3349(52.1)$ & $1574(55.2)$ & $1775(49.6)$ & \\
\hline Refusal/Don't know & $681(10.6)$ & $237(8.3)$ & $444(12.4)$ & \\
\hline \multicolumn{5}{|l|}{ Partner's sexual orientation } \\
\hline Gay or homosexual & $2487(38.7)$ & $1131(39.7)$ & $1356(37.9)$ & \\
\hline Bisexual & $3418(53.1)$ & $1532(53.7)$ & $1886(52.7)$ & $<0.001$ \\
\hline Refusal/Don't know & $527(8.2)$ & $190(6.7)$ & $337(9.4)$ & \\
\hline \multicolumn{5}{|l|}{ Partner's marital status } \\
\hline Single/Never married & $5285(82.2)$ & $2338(82.0)$ & $2947(82.3)$ & \\
\hline Married to a woman & $919(14.3)$ & $397(13.9)$ & $522(14.6)$ & \\
\hline Cohabitating with a man & $20(0.3)$ & $7(0.3)$ & $13(0.4)$ & 0.033 \\
\hline Separated/Divorced/Widowed & $39(0.6)$ & $17(0.6)$ & $22(0.6)$ & \\
\hline Refusal/Don't know & $116(1.8)$ & $69(2.4)$ & $47(1.3)$ & \\
\hline Missing & $53(0.8)$ & $25(0.88)$ & $28(0.80)$ & \\
\hline \multicolumn{5}{|l|}{ Relationship type } \\
\hline Regular & $2255(35.1)$ & $951(33.3)$ & $1304(36.4)$ & \\
\hline Casual & $4160(64.7)$ & $1890(66.3)$ & $2270(63.4)$ & 0.011 \\
\hline Refusal/Don't know & $8(0.12)$ & $6(0.2)$ & $2(0.06)$ & \\
\hline Missing & $9(0.14)$ & $6(0.2)$ & $3(0.08)$ & \\
\hline \multicolumn{5}{|l|}{ Age difference between partner and participant } \\
\hline No age difference & $548(8.5)$ & $237(8.3)$ & $311(8.7)$ & \\
\hline Age difference greater than or equal to ten years & $998(15.5)$ & $440(15.4)$ & $558(15.6)$ & $<0.001$ \\
\hline Age difference less than 10 years & $4229(65.8)$ & $1938(67.9)$ & $2291(64.0)$ & \\
\hline Unknown age difference & $657(10.2)$ & $238(8.3)$ & $419(11.7)$ & \\
\hline \multicolumn{5}{|l|}{ Partner Age mix } \\
\hline All partner's ages were different & $3264(50.8)$ & $1470(51.5)$ & $1794(50.1)$ & \\
\hline All partners were consistently younger than participant & $755(11.7)$ & $354(12.4)$ & $401(11.2)$ & \\
\hline All partners were consistently older than participant & $1976(30.7)$ & $894(31.3)$ & $1082(30.2)$ & $<0.001$ \\
\hline All partners were the same age as participant & $42(0.7)$ & $16(0.6)$ & $26(0.7)$ & \\
\hline Unknown age mix & $395(6.1)$ & $119(4.2)$ & $276(7.7)$ & \\
\hline \multicolumn{5}{|l|}{ Partner socio-economic status } \\
\hline Same as participant's & $1367(21.3)$ & $635(22.3)$ & $732(20.5)$ & \\
\hline Lower than participant's & $1386(21.6)$ & $610(21.4)$ & $776(21.7)$ & \\
\hline Higher than participant's & $3428(53.3)$ & $1520(53.3)$ & $1908(53.3)$ & 0.012 \\
\hline
\end{tabular}


Table 2 Partner and partnership characteristics by CCU with both types of anal sex (Continued)

\begin{tabular}{|c|c|c|c|}
\hline Partner and relationship characteristics & $\begin{array}{l}\text { Total } \\
(N=6432)\end{array}$ & $\begin{array}{l}\text { CCU with both types of } \\
\text { anal sex } \\
(N=2853)(44.3 \%)\end{array}$ & $\begin{array}{l}\text { Non-CCU with both types } \\
\text { of anal sex } \\
(N=3579)(55.7 \%)\end{array}$ \\
\hline Refusal/Don't know & $248(3.9)$ & $88(3.1)$ & $160(4.5)$ \\
\hline Missing & $3(0.05)$ & 0 & $3(0.08)$ \\
\hline
\end{tabular}

Did your partner have more than one regular sexual partner at the same time?

$\begin{array}{lllll}\text { No } & 963(15.0) & 448(15.7) & 515(14.4) & 2473(69.1) \\ \text { Yes } & 4403(68.5) & 1930(67.7) & 591(16.5) & 0.311\end{array}$

How many sexual partners do you believe your partner has had in the past year?

$\begin{array}{llll}\text { No sexual partners } & 104(1.6) & 44(1.5) & 60(1.7) \\ 1-2 \text { sexual partners } & 470(7.3) & 244(8.6) & 226(6.3) \\ 3-5 \text { sexual partners } & 573(8.9) & 310(10.9) & 263(7.4) \\ \text { Greater than 5 } & 589(9.2) & 309(10.9) & 280(7.8) \\ \text { Don't know } & 4696(73.0) & 1946(68.2) & 2750(76.8)\end{array}$

Do you believe partner has ever had sex under the influence of drug or alcohol?

$\begin{array}{llll}\text { No } & 2100(32.7) & 1031(36.1) & 1069(29.9) \\ \text { Yes } & 1238(19.3) & 623(21.8) & 615(17.2) \\ \text { Don't Know } & 778(12.1) & 344(12.1) & 434(12.1) \\ \text { Refusal } & 2316(36.0) & 855(30.0) & 1461(40.8)\end{array}$

When partner has sex with other sexual partners how often do you believe a condom is used

$\begin{array}{llll}\text { Never } & 237(3.7) & 82(2.9) & 155(4.3) \\ \text { Sometimes } & 1035(16.1) & 446(15.6) & 589(16.5) \\ \text { Almost always } & 296(4.6) & 124(4.4) & 172(4.8) \\ \text { Always } & 974(15.1) & 657(23.0) & 317(8.9) \\ \text { Don't know } & 1311(20.4) & 541(19.0) & 770(21.5) \\ \text { Refusal } & 2577(40.1) & 1001(35.1) & 1576(44.0) \\ \text { Missing } & 2(0.03) & 2(0.07) & 0\end{array}$

When you had sex with this partner, how often did you have sex?

$\begin{array}{llll}\text { Only once or twice ever } & 1125(17.5) & 564(19.8) & 561(15.7) \\ \text { Almost every day } & 207(3.2) & 80(2.8) & 127(3.6) \\ \text { A few times each week } & 1003(15.6) & 411(14.4) & 592(16.5) \\ \text { A few times each month } & 1478(23.0) & 651(22.8) & 827(23.1) \\ \text { Once a month } & 2598(40.4) & 1140(40.0) & 1458(40.7) \\ \text { Refusal/Don't know } & 20(0.3) & 6(0.2) & 14(0.4) \\ \text { Missing } & 1(0.02) & 1(0.04) & 0 \\ \text { As far as you know, has partner ever had any kind of STI? } & & 1223(34.2) \\ \text { No } & 2479(38.5) & 1256(44.0) & 318(8.9) \\ \text { Yes } & 551(8.6) & 233(8.2) & 2016(56.3) \\ \text { Unknown } & 3371(52.4) & 1355(47.5) & 22(0.6)\end{array}$

Has partner encouraged you to make sure you use condom when you have sex with other sexual partners?

$\begin{array}{llll}\text { No } & 2274(35.4) & 826(29.0) & 1,448(40.5) \\ \text { Yes } & 3343(52.0) & 1661(58.2) & 1682(47.0) \\ \text { Don't know } & 11(0.2) & 0(0.0) & 11(0.3) \\ \text { Refusal } & 804(12.5) & 366(12.9) & 438(12.2)\end{array}$


Table 2 Partner and partnership characteristics by CCU with both types of anal sex (Continued)

\begin{tabular}{|c|c|c|c|c|}
\hline Partner and relationship characteristics & $\begin{array}{l}\text { Total } \\
(N=6432)\end{array}$ & $\begin{array}{l}\text { CCU with both types of } \\
\text { anal sex } \\
(N=2853)(44.3 \%)\end{array}$ & $\begin{array}{l}\text { Non-CCU with both types } \\
\text { of anal sex } \\
(N=3579)(55.7 \%)\end{array}$ & $p$ value \\
\hline \multicolumn{5}{|c|}{ How frequently do you discuss HIV with partner? } \\
\hline Never & $2059(32.0)$ & $756(26.5)$ & $1303(36.4)$ & \\
\hline At least once a week & $1173(18.2)$ & $668(23.4)$ & $505(14.1)$ & \\
\hline Once a month or less & $2395(37.2)$ & $1057(37.1)$ & $1338(37.4)$ & $<0.001$ \\
\hline Refused & $805(12.5)$ & $372(13.0)$ & $433(12.1)$ & \\
\hline \multicolumn{5}{|c|}{ As far as you know what your partner's HIV status is? } \\
\hline Negative & $2598(40.4)$ & $1931(48.8)$ & $1207(33.7)$ & \\
\hline Positive & $355(5.5)$ & $127(4.5)$ & $228(6.4)$ & \\
\hline Unknown & $3460(53.8)$ & $1330(46.6)$ & $2130(59.5)$ & $<0.001$ \\
\hline Missing & $19(0.3)$ & $5(0.2)$ & $14(0.4)$ & \\
\hline \multicolumn{5}{|l|}{ HIV serostatus concordance } \\
\hline Both negative & $1363(21.2)$ & $730(25.6)$ & $633(17.7)$ & \\
\hline Both positive & $243(3.8)$ & $94(3.3)$ & $149(4.2)$ & \\
\hline HIV serodiscordant/ Unknown status & $3972(61.8)$ & $1784(62.5)$ & $2188(61.1)$ & $<0.001$ \\
\hline Both unknown & $835(13.0)$ & $240(8.4)$ & $595(16.6)$ & \\
\hline Missing & $19(0.3)$ & $23(0.4)$ & $14(0.4)$ & \\
\hline
\end{tabular}

Results for the sub-group analyses are presented as supplemental tables in the appendix. In the multivariable analysis among TGW (Supplemental Table 1), participants who reported being previously married or widowed and participants who reported that they had previously tested for HIV more than once were more likely to consistently use condoms with both types of anal sex. Conversely, transgender participants who reported their sexual orientation as other were less likely to consistently use condoms with both types of anal sex.

Findings for MSM only (Supplemental Table 3) were similar to results in for the combined analysis. In the multivariable analysis among MSM only, higher education and participants who reported that oral sex had the highest HIV transmission risk were more likely to consistently use condom while younger age group (16-24), neither buying or getting condoms for free, and MSM participants who reported being very worried about HIV in the previous 12 months were less likely to consistently use condoms with both types of anal sex.

\section{Partner and relationship characteristics associated with consistent condom use}

In the multivariable analysis (Table 4), relationships in which the partners had a higher education, casual relationships, relationships where the partner encouraged the participant to use condoms and where HIV was discussed at least once a week and larger network sizes were found to be positively associated with CCU. Older partners (35 and older), relationships where the partner was reported to be married to a woman, all frequencies of sexual acts, and relationships where the partner's HIV status were reported to be positive, or unknown were all negatively associated with CCU.

In the sub-group analyses for TGW (Supplemental Table 2), results for the multivariable analysis showed that CCU with both types of anal sex was significantly more likely where the participant's partner had an education at the secondary level or higher, or where the relationship was described as casual, while CCU was significantly less likely with any frequency of sexual acts. In the multivariable analysis among MSM (Supplemental Table 4), CCU was significantly more likely where the relationship was described as casual, where the participant reported that their partner had encouraged the participant to use condoms with their other sexual partners, and a larger network size. Consistent condom use was significantly less likely if the participant's partner was 45 or older, with any frequency of sexual acts, and if the participants reported that their partners' HIV status was positive or unknown.

\section{Discussion}

In these analyses among MSM and TGW in Nigeria, the data demonstrated on the individual level, CCU during both insertive and receptive anal sex were positively associated with higher education and disclosure of sexual behavior to a healthcare worker, and on the partnership level was associated with relationship type, partners encouraging condom use, and frequent discussion of HIV 
Table 3 Participant characteristics associated with consistent condom use with anal sex

\begin{tabular}{|c|c|c|c|c|c|c|}
\hline \multirow[t]{2}{*}{$\begin{array}{l}\text { Participant } \\
\text { characteristics }\end{array}$} & \multicolumn{2}{|c|}{ Associations with insertive anal sex } & \multicolumn{2}{|c|}{ Associations with receptive anal sex } & \multicolumn{2}{|c|}{$\begin{array}{l}\text { Associations with both types of ana } \\
\text { sex }\end{array}$} \\
\hline & $\begin{array}{l}\text { Bivariate } \\
\text { analyses (95\% } \\
\text { Cl) }\end{array}$ & $\begin{array}{l}\text { Multivariable } \\
\text { analyses }(95 \% \mathrm{Cl})\end{array}$ & $\begin{array}{l}\text { Bivariate } \\
\text { analyses (95\% } \\
\text { Cl) }\end{array}$ & $\begin{array}{l}\text { Multivariable } \\
\text { analyses }(95 \% \mathrm{Cl})\end{array}$ & $\begin{array}{l}\text { Bivariate } \\
\text { analyses (95\% } \\
\mathrm{Cl})\end{array}$ & $\begin{array}{l}\text { Multivariable } \\
\text { analyses }(95 \% \mathrm{Cl})\end{array}$ \\
\hline \multicolumn{7}{|l|}{ Age categories } \\
\hline 16-19years & Ref & Ref & Ref & Ref & Ref & Ref \\
\hline $20-24$ years & $\begin{array}{l}0.71(0.50-0.99) \\
* *\end{array}$ & $0.55(0.38-0.81)^{* *}$ & $0.94(0.71-1.24)$ & $0.76(0.56-1.03)$ & $0.89(0.70-1.15)$ & $0.75(0.57-0.99) * *$ \\
\hline 25-29years & $\begin{array}{l}0.61(0.43-0.88) \\
* *\end{array}$ & $0.41(0.28-0.62)^{* * *}$ & $0.88(0.65-1.18)$ & $0.57(0.41-0.80) * *$ & $0.79(0.60-1.03) *$ & $0.58(0.43-0.78) * *$ \\
\hline 30-34 years & $0.72(0.47-1.11)$ & $0.51(0.31-0.83) * *$ & $0.91(0.60-1.37)$ & $0.61(0.38-0.97)^{* *}$ & $0.86(0.61-1.22)$ & $0.64(0.43-0.95) * *$ \\
\hline 35 and older & $0.71(0.42-1.19)$ & $0.52(0.28-0.97) * *$ & $0.92(0.51-1.62)$ & $0.62(0.31-1.24)$ & $0.92(0.59-1.43)$ & $0.72(0.43-1.21)$ \\
\hline \multicolumn{7}{|l|}{ Education status } \\
\hline $\begin{array}{l}\text { None/primary } \\
\text { education only }\end{array}$ & Ref & Ref & Ref & Ref & Ref & Ref \\
\hline Junior secondary only & $0.87(0.44-1.71)$ & $0.76(0.36-1.60)$ & $1.15(0.61-2.12)$ & $1.03(0.52-2.05)$ & $1.32(0.75-2.33)$ & $1.25(0.68-2.28)$ \\
\hline Senior secondary & $\begin{array}{l}1.98(1.20-3.27) \\
* *\end{array}$ & $1.50(0.84-2.58)$ & $1.48(0.90-2.44)$ & $1.13(0.64-1.98)$ & $\begin{array}{l}1.82(1.17-2.82) \\
* *\end{array}$ & $1.42(0.87-2.31)$ \\
\hline $\begin{array}{l}\text { Higher than } \\
\text { secondary }\end{array}$ & $\begin{array}{l}2.02 \\
* *\end{array}(1.22-3.34)$ & $1.78(1.01-3.14)^{* *}$ & $\begin{array}{l}1.94(1.16-3.23) \\
* *\end{array}$ & $1.66(0.94-2.94)$ & $\underset{* *}{2.05}(1.31-3.21)$ & $1.80(1.10-2.94) * *$ \\
\hline Refusal/Don't know & $1.42(0.36-5.51)$ & $1.76(0.37-8.4)$ & $1.24(0.27-5.69)$ & $1.42(0.26-7.74)$ & $1.24(0.33-4.60)$ & $1.47(0.35-6.15)$ \\
\hline \multicolumn{7}{|l|}{ Sexual orientation } \\
\hline Gay or homosexual & Ref & & Ref & & Ref & \\
\hline Bisexual & $1.17(0.93-1.48)$ & & $1.04(0.85-1.27)$ & & $1.09(0.91-1.30)$ & \\
\hline Other & - & & $1.94(0.19-19.7)$ & & $1.98(0.20-20.0)$ & \\
\hline Refusal/Don't know & $4.02(0.64-25.5)$ & & $1.38(0.09-22.2)$ & & $2.95(0.42-20.8)$ & \\
\hline \multicolumn{7}{|l|}{ Gender identity } \\
\hline Male & Ref & Ref & Ref & Ref & Ref & Ref \\
\hline Female & $1.45(0.97-2.17) *$ & $1.52(0.97-2.36)$ & $1.15(0.87-1.52)$ & $1.21(0.90-1.63)$ & $1.19(0.92-1.55)$ & $1.21(0.91-1.60)$ \\
\hline Both/other & $0.82(0.57-1.17)$ & $0.81(0.56-1.18)$ & $\begin{array}{l}0.68(0.49-0.96) \\
* *\end{array}$ & $0.69(0.48-0.98) * *$ & $0.76(0.56-1.03) *$ & $0.77(0.56-1.06)$ \\
\hline Refusal/Don't know & $0.82(0.14-4.71)$ & $0.72(0.09-5.47)$ & $0.30(0.03-2.70)$ & $0.25(0.03-2.20)$ & $0.47(0.09-2.50)$ & $0.43(0.07-2.59)$ \\
\hline \multicolumn{7}{|l|}{ Marital status } \\
\hline Single/Never Married & Ref & Ref & Ref & Ref & Ref & Ref \\
\hline $\begin{array}{l}\text { Married/Cohabitating } \\
\text { with a woman }\end{array}$ & $0.99(0.64-1.52)$ & $1.39(0.84-2.32)$ & $1.05(0.66-1.68)$ & $1.28(0.75-2.17)$ & $0.99(0.66-1.47)$ & $1.27(0.81-2.00)$ \\
\hline $\begin{array}{l}\text { Cohabitating with a } \\
\text { man }\end{array}$ & $1.31(0.63-2.74)$ & $0.78(0.35-1.73)$ & $0.89(0.45-1.76)$ & $0.62(0.32-1.23)$ & $1.05(0.58-1.89)$ & $0.72(0.39-1.33)$ \\
\hline $\begin{array}{l}\text { Divorced/Separated/ } \\
\text { Widowed }\end{array}$ & $0.70(0.36-1.37)$ & $0.67(0.31-1.41)$ & $1.11(0.62-1.96)$ & $1.10(0.58-2.07)$ & $0.92(0.54-1.56)$ & $0.86(0.49-1.52)$ \\
\hline \multicolumn{7}{|c|}{ Disclosure to healthcare worker } \\
\hline No & Ref & Ref & Ref & Ref & Ref & Ref \\
\hline Yes & $1_{* * *} .65(1.33-2.05)$ & $1.31(1.03-1.66) * *$ & $\begin{array}{l}1.67(1.36-2.05) \\
* * *\end{array}$ & $1.39(1.11-1.76) * *$ & $\begin{array}{l}1.47(1.24-1.75) \\
* * *\end{array}$ & $1.22(1.00-1.48) * *$ \\
\hline Refusal/Don't know & $0.83(0.15-4.71)$ & $1.09(0.15-7.7)$ & $\begin{array}{l}0.13(0.02-0.77) \\
* *\end{array}$ & $0.09(0.02-0.43) * *$ & $0.45(0.07-2.72)$ & $0.46(0.06-3.32)$ \\
\hline \multicolumn{7}{|c|}{ Heavy Drinking in past 30 days } \\
\hline No & Ref & & Ref & & Ref & \\
\hline Yes & $1.20(0.85-1.69)$ & & $1.12(0.77-1.64)$ & & $1.12(0.82-1.52)$ & \\
\hline
\end{tabular}


Table 3 Participant characteristics associated with consistent condom use with anal sex (Continued)

\begin{tabular}{|c|c|c|c|c|c|c|}
\hline \multirow[t]{2}{*}{$\begin{array}{l}\text { Participant } \\
\text { characteristics }\end{array}$} & \multicolumn{2}{|c|}{ Associations with insertive anal sex } & \multicolumn{2}{|c|}{ Associations with receptive anal sex } & \multicolumn{2}{|c|}{$\begin{array}{l}\text { Associations with both types of ana } \\
\text { sex }\end{array}$} \\
\hline & $\begin{array}{l}\text { Bivariate } \\
\text { analyses (95\% } \\
\text { CI) }\end{array}$ & $\begin{array}{l}\text { Multivariable } \\
\text { analyses }(95 \% \mathrm{Cl})\end{array}$ & $\begin{array}{l}\text { Bivariate } \\
\text { analyses (95\% } \\
\text { Cl) }\end{array}$ & $\begin{array}{l}\text { Multivariable } \\
\text { analyses }(95 \% \mathrm{Cl})\end{array}$ & $\begin{array}{l}\text { Bivariate } \\
\text { analyses (95\% } \\
\text { Cl) }\end{array}$ & $\begin{array}{l}\text { Multivariable } \\
\text { analyses }(95 \% \mathrm{Cl})\end{array}$ \\
\hline Refusal/Don't know & $0.72(0.36-1.45)$ & & $0.81(0.38-1.76)$ & & $0.75(0.40-1.38)$ & \\
\hline \multicolumn{7}{|l|}{ Access to condoms } \\
\hline $\begin{array}{l}\text { Gets condoms for } \\
\text { free }\end{array}$ & Ref & Ref & Ref & Ref & Ref & Ref \\
\hline Buys condoms & $\begin{array}{l}0.69(0.54-0.88) \\
* *\end{array}$ & $0.82(0.631 .07)$ & $\begin{array}{l}0.74(0.58-0.94) \\
* *\end{array}$ & $0.91(0.70-1.17)$ & $\begin{array}{l}0.77(0.62-0.94) \\
* *\end{array}$ & $0.91(0.73-1.13)$ \\
\hline $\begin{array}{l}\text { Gets for free and } \\
\text { buys them }\end{array}$ & $0.87(0.67-1.13)$ & $0.81(0.61-1.06)$ & $0.90(0.70-1.15)$ & $0.92(0.71-1.20)$ & $0.88(0.71-1.09)$ & $0.86(0.68-1.07)$ \\
\hline Neither & $\underset{* *}{0.14}(0.04-0.44)$ & $0.23(0.06-0.81)^{* *}$ & $\underset{* * *}{0.15}(0.06-0.38)$ & $0.18(0.07-0.47) * * *$ & $\underset{* * *}{0.19}(0.09-0.43)$ & $0.26(0.11-0.59) * *$ \\
\hline Refusal/Don't know & $0.81(0.22-3.03)$ & $1.53(0.32-7.24)$ & $0.89(0.30-2.70)$ & $1.32(0.39-4.47)$ & $0.72(0.25-2.06)$ & $1.18(0.39-3.59)$ \\
\hline
\end{tabular}

\section{Knowledge of HIV risks}

a.) What type of sex is associated with highest HIV transmission risk?

\begin{tabular}{|c|c|c|c|c|c|c|}
\hline $\begin{array}{l}\text { All types of sex } \\
\text { carry equal risk }\end{array}$ & Ref & Ref & Ref & Ref & Ref & Ref \\
\hline Anal sex & $\begin{array}{l}1.32(1.02-1.70) \\
* *\end{array}$ & $1.15(0.88-1.50)$ & $\begin{array}{l}1.35(1.07-1.70) \\
* *\end{array}$ & $1.19(0.93-1.52)$ & $\begin{array}{l}1.31(1.07-1.60) \\
* *\end{array}$ & $1.16(0.94-1.44)$ \\
\hline Vaginal sex & $1.15(0.85-1.57)$ & $1.28(0.92-1.80)$ & $1.22(0.87-1.70)$ & $1.47(1.03-2.10) * *$ & $1.12(0.86-1.46)$ & $1.26(0.95-1.68)$ \\
\hline Oral & $1.06(0.61-1.87)$ & $1.31(0.72-2.40)$ & $1.65(0.98-2.77) *$ & $1.78(1.04-3.04) * *$ & $1.36(0.86-2.15)$ & $1.48(0.92-2.37)$ \\
\hline Refusal/Don't know & $0.76(0.42-1.39)$ & $1.20(0.63-2.27)$ & $0.82(0.47-1.44)$ & $1.11(0.61-2.03)$ & $0.75(0.45-1.23)$ & $1.04(0.61-1.76)$ \\
\hline
\end{tabular}

b.) What type of anal sex is associated with the highest HIV transmission risk?

$\begin{array}{llll}\begin{array}{l}\text { All types of anal } \\ \text { sex carry equal risk }\end{array} & \text { Ref } & \text { Ref } & \text { Ref } \\ \text { Receptive anal sex } & 1.01(0.81-1.25) & 1.14(0.93-1.40) & 1.08(0.90-1.29) \\ \text { Insertive anal sex } & 1.02(0.72-1.46) & 1.19(0.82-1.75) & 1.15(0.85-1.56) \\ \text { Refusal/Don't know } & 0.85(0.52-1.40) & 0.79(0.45-1.40) & 0.87(0.56-1.35)\end{array}$

\section{Any STI test in the past 12 months}

\begin{tabular}{|c|c|c|c|c|c|c|}
\hline No & Ref & Ref & Ref & Ref & Ref & Ref \\
\hline Yes & $\begin{array}{l}1.33 \\
* *\end{array}(1.08-1.64)$ & $1.18(0.94-1.48)$ & $\begin{array}{l}1.39 \\
* *\end{array}$ & $1.24(0.99-1.55)$ & $\begin{array}{l}1.28(1.07-1.52) \\
* *\end{array}$ & $1.14(0.94-1.37)$ \\
\hline Refusal/Don't know & $6.28(0.94-41.8)$ & $5.23(0.45-60.3)$ & $2.33(0.33-16.3)$ & $2.28(0.25-20.6)$ & $6.15(0.94-40.4)$ & $6.00(0.68-53.2)$ \\
\hline
\end{tabular}

Any STI diagnosis in the past 12 months

$\begin{array}{ll}\text { No } & \text { Ref } \\ \text { Yes } & 0.93(0.76-1.18) \\ \text { Refusal/ Don't know } & 0.53(0.13-2.09)\end{array}$

Ref

$0.84(0.67-1.06)$

$0.43(0.08-2.34)$

Worry about HIV in the past 12 months?

Not at all worried

Somewhat/ a little worried

Very worried

Refusal/Don't know
Ref

$0.94(0.74-1.20)$

$0.74(0.58-0.94)$

$0.55(0.47-0.63)$
Ref Ref

$0.83(0.70-1.08)$

$1.06(0.84-1.33)$

$0.65(0.50-0.84) * *$

$0.65(0.44-0.97)^{* *}$
0.75
$* *$
Ref

$0.88(0.72-1.07)$

$0.52(0.15-1.81)$

Ref Ref

$0.97(0.79-1.18)$

Ref

$0.95(0.74-1.21)$

$0.75(0.61-0.92)$

$0.66(0.53-0.82) * *$

$1.89(0.27-13.2) \quad 2.27(0.27-18.7)$ 
Table 3 Participant characteristics associated with consistent condom use with anal sex (Continued)

\begin{tabular}{|c|c|c|c|c|c|c|}
\hline \multirow[t]{2}{*}{$\begin{array}{l}\text { Participant } \\
\text { characteristics }\end{array}$} & \multicolumn{2}{|c|}{ Associations with insertive anal sex } & \multicolumn{2}{|c|}{ Associations with receptive anal sex } & \multicolumn{2}{|c|}{$\begin{array}{l}\text { Associations with both types of anal } \\
\text { sex }\end{array}$} \\
\hline & $\begin{array}{l}\text { Bivariate } \\
\text { analyses (95\% } \\
\text { Cl) }\end{array}$ & $\begin{array}{l}\text { Multivariable } \\
\text { analyses }(95 \% \mathrm{Cl})\end{array}$ & $\begin{array}{l}\text { Bivariate } \\
\text { analyses (95\% } \\
\text { Cl) }\end{array}$ & $\begin{array}{l}\text { Multivariable } \\
\text { analyses }(95 \% \mathrm{Cl})\end{array}$ & $\begin{array}{l}\text { Bivariate } \\
\text { analyses (95\% } \\
\text { Cl) }\end{array}$ & $\begin{array}{l}\text { Multivariable } \\
\text { analyses }(95 \% \mathrm{Cl})\end{array}$ \\
\hline \multicolumn{7}{|c|}{ Ever previously tested for HIV? } \\
\hline No & Ref & Ref & Ref & Ref & Ref & \\
\hline Yes, once & $\begin{array}{l}1.53(1.11-2.10) \\
* *\end{array}$ & $1.07(0.56-2.06)$ & $\begin{array}{l}1.59(1.19-2.13) \\
* *\end{array}$ & $1.89(1.01-3.56) * *$ & $\begin{array}{l}1.51(1.17-1.97) \\
* *\end{array}$ & $1.44(0.86-2.43)$ \\
\hline Yes, more than once & $\begin{array}{l}1.98(1.50-2.61) \\
* * *\end{array}$ & $1.36(0.70-2.67)$ & $\begin{array}{l}1.78(1.37-2.30) \\
* * *\end{array}$ & $2.02(1.05-3.89) * *$ & $\begin{array}{l}1.77(1.41-2.22) \\
* * *\end{array}$ & $1.63(0.95-2.80)$ \\
\hline Refusal/Don't know & $\begin{array}{l}13.9(2.79-68.9) \\
* *\end{array}$ & $22.1(6.68-67.5)$ & $\begin{array}{l}5.75(4.60-7.18) \\
* * *\end{array}$ & $9.72(4.93-19.2) * * *$ & $\begin{array}{l}13.2(2.68-65.4) \\
* *\end{array}$ & $20.0(6.6-60.3) * *$ \\
\hline \multicolumn{7}{|l|}{ Self-reported HIV } \\
\hline Negative & Ref & Ref & Ref & Ref & Ref & Ref \\
\hline Positive & $\begin{array}{l}1.30(1.03-1.65) \\
* *\end{array}$ & $1.18(0.91-1.54)$ & $\begin{array}{l}1.29(1.04-1.61) \\
* *\end{array}$ & $1.20(0.94-1.54)$ & $\begin{array}{l}1.22(1.01-1.48) \\
* *\end{array}$ & $1.13(0.91-1.39)$ \\
\hline Unknown & $\begin{array}{l}0.62(0.48-0.81) \\
* * *\end{array}$ & $0.82(0.44-1.54)$ & $\begin{array}{l}0.72(0.56-0.92) \\
* *\end{array}$ & $1.53(0.83-2.84)$ & $\begin{array}{l}0.68(0.55-0.85) \\
* *\end{array}$ & $1.10(0.67-1.82)$ \\
\hline \multicolumn{7}{|l|}{ HIV results at baseline } \\
\hline Negative & Ref & & Ref & & Ref & \\
\hline Positive & $0.90(0.73-1.10)$ & & $0.89(0.73-1.08)$ & & $0.90(0.76-1.06)$ & \\
\hline \multicolumn{7}{|l|}{ Site } \\
\hline Abuja & Ref & Ref & Ref & Ref & Ref & Ref \\
\hline Lagos & $\begin{array}{l}2.03(1.62-2.54) \\
* * *\end{array}$ & $1.76(1.36-2.29) * * *$ & $\begin{array}{l}1.47(1.21-1.79) \\
* * *\end{array}$ & $1.21(0.95-1.54)$ & $\begin{array}{l}1.60(1.34-1.89) \\
* * *\end{array}$ & $1.41(1.15-1.73) * *$ \\
\hline
\end{tabular}

by partners. Given that consistent condom use was reported in only $44 \%$ of the partnerships reported here and the prevalence of HIV remains high among MSM and TGW in Nigeria, our results highlight possible correlates of condom use which may be leveraged for HIV prevention messaging.

The findings that condom use was more likely in relationships where condom use was encouraged and in relationships where HIV was discussed frequently is consistent with previous studies which highlight that relationship and partner norms influence health behaviors, including condom use $[16,20,30]$. In a study among MSM in the US, it was found that having at least one person in a sexual dyad who did not disapprove of condomless anal sex was associated with an increased likelihood of condomless sex compared to those who did not have such a person in their sexual network [36]. Relationships present opportunities for information exchange and involved parties often influence each other leading to sustained behavior change. This dynamic could be leveraged to create interventions that promote open discussion of safer sex practices. Such interventions may require training for effective communication, as openly discussing sexual behaviors may be uncommon in some communities, particularly ones where HIV and same-sex sexual practices are heavily stigmatized $[9,37]$.

Results showing that CCU was more likely in casual relationships as compared to regular relationships is also consistent with findings from other studies among MSM where higher rates of unprotected anal intercourse (UAI) have been observed in relationships considered more serious [17, 23, 31]. In these relationships, couples may engage in UAI to increase intimacy and trust in a relationship [15]. While risk perception may decrease with more regular partners, there may be sustained risks for both STIs and HIV in non-exclusive relationships, therefore the importance of prevention modalities should be emphasized for MSM or TGW in non-exclusive relationships. Moreover, previous studies have associated steady partnership as a source of HIV infection among gay men [11, 12]. These findings highlight both the importance and the potential of couple-oriented testing and counseling practices. Couple-based approaches have been shown to be highly effective in several settings and evaluation in the Nigerian context for MSM and TGW may be valuable $[14,38]$. 
Table 4 Partner and relationship characteristics associated with consistent condom use with anal sex

\begin{tabular}{|c|c|c|c|c|c|c|}
\hline \multirow[t]{2}{*}{$\begin{array}{l}\text { Partner and relationship } \\
\text { characteristics }\end{array}$} & \multicolumn{2}{|c|}{ Associations with insertive anal sex } & \multicolumn{2}{|c|}{ Associations with receptive anal sex } & \multicolumn{2}{|c|}{$\begin{array}{l}\text { Associations with both types of anal } \\
\text { sex }\end{array}$} \\
\hline & $\begin{array}{l}\text { Bivariate } \\
\text { analyses } \\
(95 \% \mathrm{Cl})\end{array}$ & $\begin{array}{l}\text { Multivariable } \\
\text { analyses }(95 \% \mathrm{Cl})\end{array}$ & $\begin{array}{l}\text { Bivariate } \\
\text { analyses } \\
(95 \% \mathrm{Cl})\end{array}$ & $\begin{array}{l}\text { Multivariable } \\
\text { analyses }(95 \% \mathrm{Cl})\end{array}$ & $\begin{array}{l}\text { Bivariate } \\
\text { analyses } \\
(95 \% \mathrm{Cl})\end{array}$ & $\begin{array}{l}\text { Multivariable } \\
\text { analyses }(95 \% \mathrm{CI})\end{array}$ \\
\hline \multicolumn{7}{|l|}{ Partner age categories } \\
\hline Younger than 25 & Ref & Ref & Ref & Ref & Ref & Ref \\
\hline $25-34$ & $0.96(0.87-1.05)$ & $0.93(0.84-1.03)$ & $1.03(0.92-1.14)$ & $0.94(0.84-1.06)$ & $1.00(0.92-1.09)$ & $0.95(0.87-1.05)$ \\
\hline $35-44$ & $0.92(0.79-1.07)$ & $0.87(0.72-1.05)$ & $0.98(0.82-1.16)$ & $0.84(0.68-1.03)$ & $0.91(0.79-1.06)$ & $0.84(0.70-0.99) * *$ \\
\hline 45 and older & $0.94(0.72-1.22)$ & $0.80(0.59-1.08)$ & $0.77(0.57-1.05) *$ & $0.62(0.42-0.90) * *$ & $0.81(0.61-1.07)$ & $0.69(0.50-0.96) * *$ \\
\hline Refusal/Don't know & $0.97(0.78-1.21)$ & $0.27(0.05-1.47)$ & $0.85(0.68-1.06)$ & $0.59(0.16-2.24)$ & $0.89(0.74-1.07)$ & $0.56(0.17-1.90)$ \\
\hline \multicolumn{7}{|l|}{ Partner's education status } \\
\hline None/Primary education only & Ref & Ref & Ref & Ref & Ref & Ref \\
\hline Junior secondary only & $0.96(0.72-1.28)$ & $1.01(0.75-1.36)$ & $0.91(0.64-1.29)$ & $0.85(0.59-1.22)$ & $0.91(0.67-1.25)$ & $0.91(0.66-1.25)$ \\
\hline Senior secondary & $1.15(0.89-1.49)$ & $1.21(0.92-1.59)$ & $1.24(0.96-1.59) *$ & $1.27(0.98-1.65)$ & $1.19(0.95-1.48)$ & $1.24(0.99-1.57)$ \\
\hline Higher than senior secondary & $1.10(0.85-1.43)$ & $1.22(0.93-1.59)$ & $1.43(1.10-1.85) * *$ & $1.48(1.13-1.94) * *$ & $1.25(1.00-1.57) * *$ & $1.36(1.07-1.72) * *$ \\
\hline Refusal/Don't know & $1.18(0.86-1.62)$ & $1.28(0.93-1.78)$ & $1.24(0.91-1.69)$ & $1.33(0.97-1.83)$ & $1.19(0.91-1.56)$ & $1.28(0.97-1.70)$ \\
\hline \multicolumn{7}{|l|}{ Partner's sexual orientation } \\
\hline Gay or homosexual & Ref & & Ref & & Ref & \\
\hline Bisexual & $0.99(0.90-1.08)$ & & $1.07(0.96-1.19)$ & & $0.99(0.90-1.08)$ & \\
\hline Refusal/Don't know & $1.02(0.84-1.25)$ & & $0.93(0.72-1.21)$ & & $0.91(0.74-1.12)$ & \\
\hline \multicolumn{7}{|l|}{ Partner's marital status } \\
\hline Single/never married & Ref & Ref & Ref & Ref & Ref & Ref \\
\hline Married to a woman & $1.01(0.92-1.12)$ & $1.04(0.92-1.18)$ & $0.96(0.85-1.10)$ & $0.91(0.78-1.05)$ & $0.95(0.86-1.06)$ & $0.95(0.84-1.08)$ \\
\hline Cohabitating with a man & $0.61(0.13-2.83)$ & $0.61(0.15-2.53)$ & $0.32(0.08-1.19) *$ & $0.33(0.11-0.98) * *$ & $0.32(0.09-1.11) *$ & $0.33(0.11-0.97)^{* *}$ \\
\hline Separated/divorced/widowed & $1.02(0.74-1.41)$ & $1.08(0.78-1.50)$ & $0.95(0.60-1.51)$ & $0.93(0.58-1.48)$ & $1.06(0.69-1.65)$ & $1.09(0.71-1.68)$ \\
\hline Refusal/Don't know & $1.29(0.96-1.75) *$ & $1.50(1.09-2.07) * *$ & $1.14(0.79-1.63)$ & $1.38(0.93-2.03)$ & $1.34(0.98-1.82) *$ & $1.66(1.19-2.33) * *$ \\
\hline \multicolumn{7}{|l|}{ Relationship type } \\
\hline Regular & Ref & Ref & Ref & Ref & Ref & Ref \\
\hline Casual & $\begin{array}{l}1.23(1.13-1.33) \\
* * *\end{array}$ & $1.13(1.02-1.24) * *$ & $\begin{array}{l}1.29(1.18-1.41) \\
* * *\end{array}$ & $\begin{array}{l}1.23(1.10-1.38) \\
* * *\end{array}$ & $\begin{array}{l}1.31(1.21-1.41) \\
* * *\end{array}$ & $\begin{array}{l}1.22(1.11-1.34) \\
* * *\end{array}$ \\
\hline Refusal/Don't know & $0.55(0.08-3.93)$ & $0.52(0.07-3.68)$ & $1.29(0.74-2.27)$ & $0.98(0.71-1.34)$ & $0.75(0.11-5.12)$ & $0.69(0.08-5.90)$ \\
\hline \multicolumn{7}{|l|}{ Age Difference between partner and participant } \\
\hline No age difference & Ref & Ref & Ref & Ref & Ref & Ref \\
\hline Greater than or equal to ten years & $0.97(0.85-1.12)$ & $1.01(0.86-1.18)$ & $1.11(0.94-1.31)$ & $1.24(1.02-1.51)^{* *}$ & $1.02(0.89-1.18)$ & $1.11(0.95-1.31)$ \\
\hline Less than ten years & $0.95(0.85-1.05)$ & $0.95(0.85-1.06)$ & $1.06(0.93-1.21)$ & $1.08(0.93-1.25)$ & $1.00(0.90-1.11)$ & $1.00(0.90-1.13)$ \\
\hline Unknown age difference & $0.96(0.75-1.21)$ & $3.69(0.67-20.3)$ & $0.89(0.70-1.14)$ & $1.63(0.42-6.35)$ & $0.90(0.74-1.10)$ & $1.70(0.49-5.88)$ \\
\hline \multicolumn{7}{|l|}{ Partner Age mix } \\
\hline All partner's ages were different & Ref & Ref & Ref & Ref & Ref & Ref \\
\hline $\begin{array}{l}\text { All partners were consistently younger than } \\
\text { participant }\end{array}$ & $0.99(0.75-1.31)$ & $1.05(0.78-1.40)$ & $0.87(0.60-1.25)$ & $0.85(0.58-1.23)$ & $1.05(0.82-1.36)$ & $1.06(0.81-1.38)$ \\
\hline All partners were consistently older than participant & $1.01(0.80-1.28)$ & $1.11(0.87-1.42)$ & $0.96(0.78-1.19)$ & $0.92(0.74-1.15)$ & $0.99(0.82-1.19)$ & $0.99(0.82-1.20)$ \\
\hline All partners were the same age as participant & $0.66(0.24-1.83)$ & $0.66(0.24-1.80)$ & $2.15(0.78-5.88)$ & $2.54(0.93-6.9)$ & $1.05(0.47-2.32)$ & $1.09(0.49-2.42)$ \\
\hline Unknown age mix & $0.53(0.33-0.85) * *$ & $0.57(0.32-1.02)$ & $0.50(0.31-0.79) * *$ & $0.59(0.33-1.03)$ & $0.56(0.38-0.84) * *$ & $0.63(0.39-1.03)$ \\
\hline \multicolumn{7}{|l|}{ Partner socio-economic status } \\
\hline Same as participant's & Ref & Ref & Ref & Ref & Ref & Ref \\
\hline Lower than participant's & $1.01(0.88-1.15)$ & $0.98(0.85-1.14)$ & $0.97(0.83-1.14)$ & $0.96(0.81-1.15)$ & $1.02(0.89-1.16)$ & $0.99(0.83-1.11)$ \\
\hline Higher than participant's & $0.95(0.84-1.07)$ & $0.96(0.84-1.09)$ & $1.08(0.93-1.25)$ & $1.09(0.94-1.28)$ & $1.00(0.89-1.13)$ & $1.02(0.90-1.16)$ \\
\hline Refusal/don't know & $1.00(0.71-1.42)$ & $1.09(0.76-1.57)$ & $0.71(0.51-1.00) *$ & $0.85(0.59-1.23)$ & $0.90(0.67-1.22)$ & $0.99(0.72-1.37)$ \\
\hline \multicolumn{7}{|c|}{ Did your partner have more than one regular sexual partner at the same time? } \\
\hline No & Ref & & Ref & & Ref & \\
\hline Yes & $1.14(0.96-1.36)$ & & $1.07(0.91-1.26)$ & & $1.09(0.96-1.25)$ & \\
\hline Don't know & $1.16(0.89-1.51)$ & & $1.16(0.90-1.50)$ & & $1.10(0.88-1.36)$ & \\
\hline
\end{tabular}


Table 4 Partner and relationship characteristics associated with consistent condom use with anal sex (Continued)

\begin{tabular}{|c|c|c|c|c|c|c|}
\hline \multirow[t]{2}{*}{$\begin{array}{l}\text { Partner and relationship } \\
\text { characteristics }\end{array}$} & \multicolumn{2}{|c|}{ Associations with insertive anal sex } & \multicolumn{2}{|c|}{ Associations with receptive anal sex } & \multicolumn{2}{|c|}{$\begin{array}{l}\text { Associations with both types of ana } \\
\text { sex }\end{array}$} \\
\hline & $\begin{array}{l}\text { Bivariate } \\
\text { analyses } \\
(95 \% \mathrm{Cl})\end{array}$ & $\begin{array}{l}\text { Multivariable } \\
\text { analyses }(95 \% \mathrm{Cl})\end{array}$ & $\begin{array}{l}\text { Bivariate } \\
\text { analyses } \\
(95 \% \mathrm{Cl})\end{array}$ & $\begin{array}{l}\text { Multivariable } \\
\text { analyses }(95 \% \mathrm{Cl})\end{array}$ & $\begin{array}{l}\text { Bivariate } \\
\text { analyses } \\
(95 \% \mathrm{Cl})\end{array}$ & $\begin{array}{l}\text { Multivariable } \\
\text { analyses }(95 \% \mathrm{CI}\end{array}$ \\
\hline \multicolumn{7}{|c|}{ How many sexual partners do you believe your partner has had in the past year? } \\
\hline No other sexual partners & Ref & Ref & Ref & Ref & Ref & Ref \\
\hline $1-2$ sexual partners & $1.11(0.74-1.66)$ & $1.10(0.74-1.63)$ & $1.39(0.95-2.03) *$ & $1.32(0.90-1.93)$ & $1.24(0.86-1.80)$ & $1.19(0.81-1.73)$ \\
\hline $3-5$ sexual partners & $1.40(0.97-2.02) *$ & $1.43(1.00-2.06)$ & $1.61(1.13-2.30) * *$ & $1.54(1.08-2.19) * *$ & $1.43(1.00-2.03) *$ & $1.37(0.96-1.96)$ \\
\hline Greater than 5 & $1.63(1.09-2.43) * *$ & $1.66(1.11-2.49) * *$ & $1.36(0.92-1.99)$ & $1.32(0.89-1.96)$ & $1.36(0.04-1.98)$ & $1.34(0.91-1.98)$ \\
\hline Don't know & $1.28(0.90-1.83)$ & $1.49(1.04-2.14)^{* *}$ & $1.32(0.93-1.87)$ & $1.45(1.01-2.09)^{* *}$ & $1.27(0.90-1.79)$ & $1.37(0.95-1.96)$ \\
\hline
\end{tabular}

Do you believe partner has ever had sex under the influence of drug or alcohol?

$\begin{array}{lllllll}\text { No } & \text { Ref } & \text { Ref } & \text { Ref } & \text { Ref } & \text { Ref } & \text { Ref } \\ \text { Yes } & 1.08(0.92-1.28) & 1.06(0.89-1.26) & 0.91(0.78-1.06) & 0.92(0.78-1.08) & 0.96(0.84-1.11) & 0.96(0.83-1.11) \\ \text { Don't know } & 0.94(0.71-1.25) & 0.90(0.67-1.20) & 0.87(0.67-1.13) & 0.89(0.67-1.18) & 0.88(0.70-1.12) & 0.87(0.68-1.11) \\ \text { Refusal } & 0.59(0.47-0.73) & 0.45(0.29-0.71) & 0.60(0.48-0.74) & 0.56(0.43-0.75) & 0.65(0.54-0.78) & 0.60(0.41-0.89) * * \\ & * * * * * * * & * * * & * * *\end{array}$

When partner has sex with other sexual partners how often do you believe a condom is used

$\begin{array}{llll}\text { Never } & \text { Ref } & \text { Ref } & \text { Ref } \\ \text { Sometimes } & 0.73(0.48-1.10) & 0.63(0.42-0.95) * * & 1.05(0.74-1.48) \\ \text { Almost always } & 0.85(0.52-1.38) & 0.73(0.44-1.21) & 0.81(0.53-1.23) \\ \text { Always } & 0.98(0.65-1.46) & 0.88(0.59-1.32) & 1.22(0.87-1.71) \\ \text { Don't know } & 0.86(0.56-1.31) & 0.82(0.53-1.27) & 0.99(0.68-1.44) \\ \text { Refusal } & 0.70(0.47-1.03) * & 0.95(0.58-1.55) & 0.79(0.55-1.15)\end{array}$

$\begin{array}{ll}\text { Ref } & \text { Ref } \\ 1.05(0.78-1.41) & 0.94(0.70-1.27) \\ 0.89(0.62-1.28) & 0.82(0.57-1.17) \\ 1.29(0.97-1.73) * & 1.17(0.87-1.57) \\ 1.08(0.79-1.49) & 1.12(0.81-1.55) \\ 0.92(0.68-1.25) & 1.03(0.68-1.58)\end{array}$

When you had sex with this partner, how often did you have sex?

\begin{tabular}{|c|c|c|c|c|c|c|}
\hline Only once or twice ever & Ref & Ref & Ref & Ref & Ref & Ref \\
\hline Almost every day & $\begin{array}{l}0.64 \\
* * *\end{array}$ & $0.68(0.52-0.87) * *$ & $\begin{array}{l}0.63(0.50-0.80) \\
* * *\end{array}$ & $0.71(0.54-0.92)^{* *}$ & $\begin{array}{l}0.57(0.45-0.71) \\
* * *\end{array}$ & $0.64(0.50-0.82) * * *$ \\
\hline A few times each week & $\underset{* * *}{0.69}(0.57-0.83)$ & $0.73(0.60-0.89) * *$ & $\begin{array}{l}0.63(0.52-0.76) \\
* * *\end{array}$ & $0.70(0.57-0.87)^{* *}$ & $\begin{array}{l}0.59(0.50-0.69) \\
* * *\end{array}$ & $0.66(0.55-0.79) * * *$ \\
\hline A few times each month & $0.76(0.65-0.90) * *$ & $0.77(0.65-0.92) * *$ & $\begin{array}{l}0.68(0.57-0.82) \\
* * *\end{array}$ & $\begin{array}{l}0.71(0.59-0.85) \\
* * *\end{array}$ & $\begin{array}{l}0.64(0.56-0.75) \\
* * *\end{array}$ & $0.67(0.57-0.78) * * *$ \\
\hline Once a month & $0.83(0.71-0.96)^{*}$ & $0.81(0.69-0.95)^{* *}$ & $\begin{array}{l}0.72(0.61-0.84) \\
* * *\end{array}$ & $\begin{array}{l}0.70(0.60-0.83) \\
* * *\end{array}$ & $\underset{* * *}{0.70}(0.62-0.80)$ & $0.69(0.60-0.80) * * *$ \\
\hline Refusal/don't know & $0.66(0.41-1.01)$ & $0.59(0.34-1.03)$ & $0.85(0.63-1.14)$ & $1.04(0.73-1.48)$ & $0.56(0.36-0.86) * *$ & $0.58(0.37-0.90) * *$ \\
\hline \multicolumn{7}{|c|}{ As far as you know, has partner ever had any kind of STI? } \\
\hline No & Ref & Ref & Ref & Ref & Ref & Ref \\
\hline Yes & $0.79(0.65-0.96)^{* *}$ & $0.88(0.72-1.08)$ & $0.73(0.59-0.90) * *$ & $0.95(0.75-1.20)$ & $0.78(0.66-0.92)$ & $0.94(0.79-1.12)$ \\
\hline Unknown & $0.84(0.70-1.01) *$ & $0.95(0.78-1.16)$ & $0.84(0.70-1.01) *$ & $1.09(0.87-1.36)$ & $0.86(0.74-1.00) * *$ & $1.03(0.86-1.23)$ \\
\hline \multicolumn{7}{|c|}{ Tas partner encouraged you to make sure you use condom when you have sex with other sexual partners? } \\
\hline No & Ref & Ref & Ref & Ref & Ref & Ref \\
\hline Yes & $1.15(1.02-1.31)^{* *}$ & $1.09(0.96-1.24)$ & $1.15(1.02-1.31)^{* *}$ & $1.13(0.99-1.20)$ & $1.20(1.07-1.33)^{* *}$ & $1.14(1.02-1.28)^{* *}$ \\
\hline Don't know & - & - & - & - & - & - \\
\hline Refusal & $1.28(1.09-1.51) * *$ & $1.00(0.73-1.36)$ & $1.18(0.97-1.45)$ & $1.20(0.91-1.58)$ & $1.31(1.12-1.53) * *$ & $1.04(0.74-1.45)$ \\
\hline \multicolumn{7}{|c|}{ How frequently do you discuss HIV with partner? } \\
\hline Never & Ref & & Ref & Ref & Ref & Ref \\
\hline At least once a week & $1.25(1.02-1.53) * *$ & $1.17(0.94-1.45)$ & $1.33(1.10-1.62) * *$ & $1.24(1.01-1.52) * *$ & $1.31(1.11-1.54) * *$ & $1.20(1.01-1.43) * *$ \\
\hline Once a month or less & $1.26(1.08-1.47) * *$ & $1.24(1.05-1.46) * *$ & $1.03(0.88-1.22)$ & $0.99(0.84-1.18)$ & $1.12(0.98-1.29) *$ & $1.09(0.95-1.25)$ \\
\hline Refused & $1.36(1.15-1.61)$ & $1.31(0.97-1.77)$ & $1.17(0.97-1.42)$ & $1.05(0.83-1.35)$ & $1.31(1.12-1.53) * *$ & $1.29(0.94-1.76)$ \\
\hline
\end{tabular}


Table 4 Partner and relationship characteristics associated with consistent condom use with anal sex (Continued)

\begin{tabular}{|c|c|c|c|c|c|c|}
\hline \multirow[t]{2}{*}{$\begin{array}{l}\text { Partner and relationship } \\
\text { characteristics }\end{array}$} & \multicolumn{2}{|c|}{ Associations with insertive anal sex } & \multicolumn{2}{|c|}{ Associations with receptive anal sex } & \multicolumn{2}{|c|}{$\begin{array}{l}\text { Associations with both types of anal } \\
\text { sex }\end{array}$} \\
\hline & $\begin{array}{l}\text { Bivariate } \\
\text { analyses } \\
(95 \% \mathrm{Cl})\end{array}$ & $\begin{array}{l}\text { Multivariable } \\
\text { analyses }(95 \% \mathrm{Cl})\end{array}$ & $\begin{array}{l}\text { Bivariate } \\
\text { analyses } \\
(95 \% \mathrm{Cl})\end{array}$ & $\begin{array}{l}\begin{array}{l}\text { Multivariable } \\
\text { analyses }(95 \% \mathrm{Cl})\end{array}\end{array}$ & $\begin{array}{l}\text { Bivariate } \\
\text { analyses } \\
(95 \% \mathrm{Cl})\end{array}$ & $\begin{array}{l}\text { Multivariable } \\
\text { analyses }(95 \% \mathrm{Cl})\end{array}$ \\
\hline \multicolumn{7}{|c|}{ As far as you know what your partner's HIV status is? } \\
\hline Negative & Ref & & Ref & Ref & Ref & Ref \\
\hline Positive & $0.68(0.53-0.88) * *$ & $0.67(0.44-1.03)$ & $\underset{* * *}{0.42}(0.31-0.57)$ & $0.39(0.22-0.70)^{* *}$ & $0.50(0.39-0.63)$ & $0.43(0.27-0.68) * * *$ \\
\hline Unknown & $0.77(0.65-0.92) * *$ & $0.79(0.60-1.04)$ & $\begin{array}{l}0.67(0.56-0.80) \\
* * *\end{array}$ & $0.75(0.56-1.00)$ & $\begin{array}{l}0.71(0.61-0.83) \\
* * *\end{array}$ & $0.74(0.58-0.94) * *$ \\
\hline \multicolumn{7}{|l|}{ HIV serostatus concordance } \\
\hline Both negative & Ref & Ref & Ref & Ref & Ref & Ref \\
\hline Both positive & $0.68(0.47-0.97)^{* *}$ & $1.07(0.59-1.95)$ & $\underset{* * *}{0.40}(0.27-0.60)$ & $1.22(0.61-2.45)$ & $\begin{array}{l}0.51(0.37-0.71) \\
* * *\end{array}$ & $1.39(0.78-2.49)$ \\
\hline HIV serodiscordant/ unknown status & $0.87(0.70-1.07)$ & $0.99(0.74-1.33)$ & $0.72(0.57-0.90) * *$ & $0.93(0.69-1.23)$ & $0.80(0.67-0.96) * *$ & $1.02(0.80-1.30)$ \\
\hline Both unknown & $0.56(0.40-0.78) * *$ & $0.68(0.44-1.07)$ & $0.44(0.32-0.61)$ & $0.62(0.40-0.97) * *$ & $\begin{array}{l}0.50(0.38-0.66) \\
* * *\end{array}$ & $0.71(0.48-1.04)$ \\
\hline \multicolumn{7}{|l|}{ Network size } \\
\hline Smaller & Ref & Ref & Ref & Ref & Ref & Ref \\
\hline Larger & $1.41(1.13-1.76)^{* *}$ & $1.47(1.16-1.84) * *$ & $1.40(1.13-1.75) * *$ & $\begin{array}{l}1.51(1.21-1.90) \\
* * *\end{array}$ & $1.42(1.17-1.71) * *$ & $1.48(1.22-1.80) * * *$ \\
\hline Unknown & $0.99(0.71-1.37)$ & $1.19(0.83-1.71)$ & $1.03(0.74-1.42)$ & $1.28(0.90-1.84)$ & $1.15(0.89-1.50)$ & $1.34(1.00-1.79)$ \\
\hline
\end{tabular}

\section{${ }^{*} p \leq 0.10$}

** $p<0.05$

*** $p<0.001$

Our results also show that participants in relationships where the partners were married to women were less likely to consistently use condoms. The majority of participants in this study reported that they were bisexual and marriage to women was common among their partners. Given the presence of punitive laws prohibiting same sexrelationships in Nigeria, this is unsurprising [37]. Many individuals with gay or bisexual identities may choose to marry women due to fear of stigma, societal pressures, or to avoid being outed [34]. Previous surveys of men who have sex with men and women (MSMW) have shown them to have riskier HIV-related behaviors that potentiate HIV infection, including a higher probability of having an unknown HIV status and a lower probability of encountering HIV prevention activities and materials due to presence of "biphobia" [25]. Thus, MSMW could play a potential role in HIV transmission to women by bridging the epidemics in two different populations. This dynamic could also apply in TGW who have sex with cisgender women. These results suggest that addressing the prevention needs of MSMW and TGW and all their sex partners is needed for HIV reduction among both KP groups and women. Tailored prevention strategies requires context and understanding of behaviors and sexual networks of TGW who have sex with women and MSMW.

Unlike findings from other studies that have shown age-disparate relationships to be associated with more high-risk HIV-related behaviors including unprotected anal intercourse, our results showed increased odds of condom use during receptive anal sex in relationships where there was a ten year or more difference in age between partners. The risks associated with age-disparate partnerships are often higher in relationships where adolescents have partners who are much older than them. This is due to the increased likelihood for older partners to be living with HIV and a high propensity for condomless sex and alcohol use during sex with older partners $[4,19]$. There is also evidence that it may be linked to power dynamics in the relationships [27]. This could lead to a limited ability on the part of younger MSM to adopt or advocate for HIV risk reduction behaviors like condom use even when protected sex is desired. Thus, a ten-year age difference between two middle-aged persons may not be associated with the same risk as a tenyear age difference between an adolescent and a middleaged person; a nuance which is not sufficiently captured in this analysis and may therefore be the reason for the difference in the results observed.

On the individual level, disclosure of sexual behavior to a healthcare practitioner was also positively associated with CCU. Disclosure of key-population status may indicate more engagement with healthcare services and therefore reduced likelihood for risky behaviors due to more access to targeted HIV prevention messaging. Disclosure of MSM status in Nigeria may be challenged by the presence of laws criminalizing same sex behaviors and the high levels of stigma faced by MSM in the 
country $[9,37]$. Studies have demonstrated that structural elements, where the risks of disclosure of sexual practices for a meaningful risk assessment outweigh the potential benefits of better engagement in HIV prevention services, have affected the uptake of HIV prevention services including condom uptake among MSM at risk for HIV acquisition [9,37]. This is particularly important as our results showed that participants who reported neither buying condoms nor getting condoms for free were significantly less likely to consistently use condoms, a prevention modality commonly available for free in healthcare centers. Given the importance of the healthcare sector in managing and reducing the HIV epidemic among MSM in the country, training and sensitizing healthcare workers about the impacts of stigmatizing behavior is needed. De-centralization of HIV prevention services by leveraging KP community-engaged facilities such as the trusted community health centers involved in this study may also increase healthcare engagement and alter HIV-related risk behaviors. Longitudinal analysis of data from this cohort showed an improvement in the uptake of condoms and water-based lubricants by MSM and TGW, suggesting effectiveness of these facilities in improving engagement in care [8].

The findings from this study should be interpreted in the context of several limitations. Firstly, all behavioral data collected, including partner and relationship characteristics, were self-reported by the participant in the study. This makes our findings susceptible to multiple biases, including recall and social desirability. The presence of missing data may have biased our results however, missingness was around $1 \%$ for many of the variables and is unlikely to significantly impact our interpretation. Additionally, "refusal" and "don't know" categories were included in models where applicable as these responses may be informative about behavioral risks. Although the study was longitudinal, we carried out cross-sectional analyses using the baseline data. Thus, causality cannot be inferred from our results. While our results may capture the individual and relationship characteristics of MSM and TGW in Lagos and Abuja, Nigeria, our findings may not be generalizable to the broader key population in Nigeria or other countries in sub-Saharan Africa.

\section{Conclusions}

HIV prevention programs may benefit from encouraging open communication of safer sex practices as a strategy for HIV risk reduction given that MSM and TGW were more likely to consistently use condoms when their partners encouraged and openly discussed safer sex. Even with the introduction and scale up of other biomedical HIV prevention modalities such as PrEP, condom use remains an important mode for reducing onward transmission of STIs as HIV and STI infection rates remains sustained among MSM. Implementation science research could support the evaluation of optimal strategies that integrate open discussions about safe sex into existing behavioral interventions. However, while research is helpful, leveraging existing strategies to increase condom use including the provision of condoms in safe spaces, ensuring access to better condoms and condom-compatible lubricants, and teaching condom negotiation skills may support increased consistent condom use among men who have sex with men and transgender women across Nigeria.

\section{Abbreviations}

AOR: Adjusted odds ratio; CCU: Consistent condom use; Cl: Confidence interval; HIV: Human immunodeficiency virus; IQR: Interquartile range; MSM: Men who have sex with men; MSMW: Men who have sex with men and women; N: Number; OR: Odds ratio; P: $p$-value; PrEP: Pre-exposure prophylaxis; RDS: Respondent-driven sampling; SSA: Sub-Saharan Africa; STI: Sexually transmitted infection; TGW: Transgender women;

UAl: Unprotected anal intercourse

\section{Supplementary Information}

The online version contains supplementary material available at https://doi. org/10.1186/s12889-021-11275-w.

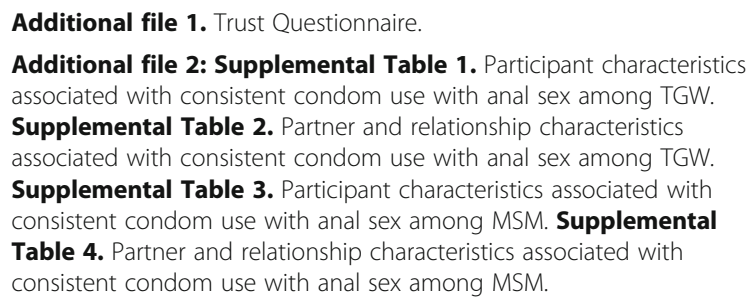

\section{Acknowledgements}

The TRUST/RV368 Study Group includes Principal Investigators: Manhattan Charurat (IHV, University of Maryland, Baltimore, MD, USA), Julie Ake (MHRP, Walter Reed Army Institute of Research, Silver Spring, MD, USA); CoInvestigators: Aka Abayomi, Sylvia Adebajo, Stefan Baral, Trevor Crowell, Charlotte Gaydos, Afoke Kokogho, Jennifer Malia, Olumide Makanjuola, Nelson Michael, Nicaise Ndembi, Rebecca Nowak, Oluwasolape Olawore, Zahra Parker, Sheila Peel, Habib Ramadhani, Merlin Robb, Cristina Rodriguez-Hart, Eric Sanders-Buell, Elizabeth Shoyemi, Sodsai Tovanabutra, Sandhya Vasan; Institutions: Institute of Human Virology at the University of Maryland School of Medicine (IHV-UMB), Johns Hopkins Bloomberg School of Public Health (JHSPH), Johns Hopkins University School of Medicine (JHUSOM), U.S. Military HIV Research Program (MHRP), Walter Reed Army Institute of Research (WRAI R), Henry M. Jackson Foundation for the Advancement of Military Medicine (HJF), Henry M. Jackson Foundation Medical Research International (HJFMRI), Institute of Human Virology Nigeria (IHVN), International Centre for Advocacy for the Right to Health (ICARH), The Initiative for Equal Rights (TIERS), Population Council Nigeria.

\section{Disclaimer}

This work was presented, in part, at the international meeting of the Institute of Human Virology 2018 in Baltimore, MD, 22-25 October 2018.

\section{Authors' contributions}

$\mathrm{OO}$ designed the analysis and authored the first draft of the manuscript. AK, $\mathrm{SA}, \mathrm{RN}$, and HR oversaw data collection and laboratory testing. OO, TC, JA, $\mathrm{SK}, \mathrm{HR}, \mathrm{HL}, \mathrm{RN}, \mathrm{MC}$, and $\mathrm{SB}$ contributed to the design of the analysis and assisted in the interpretation of results. All authors reviewed this manuscript, provided feedback, and approved of the manuscript in its final form. 


\section{Funding}

Support for the development and implementation of the TRUST/RV368 study, and collecting, analyzing and interpreting the data, and writing of the manuscript was provided by a cooperative agreement between the Henry M. Jackson Foundation for the Advancement of Military Medicine, Inc., and the U.S. Department of Defense [W81XWH-11-2-0174, W81XWH-18-2-0040]; the National Institutes of Health [R01 MH099001, R01 Al120913, R01 MH110358]; Fogarty Epidemiology Research Training for Public Health Impact in Nigeria program [D43TW010051]; and the President's Emergency Plan for AIDS Relief through a cooperative agreement between the Department of Health and Human Services/Centers for Disease Control and Prevention, Global AIDS Program, and the Institute for Human Virology-Nigeria [NU2GGH002099]. The views expressed are those of the authors and should not be construed to represent the positions of the U.S. Army, the Department of Defense, or the Department of Health and Human Services. The investigators have adhered to the policies for protection of human subjects as prescribed in AR-70.

\section{Availability of data and materials}

In order to protect the clinic staff and the participants from stigma and criminalization of same sex behavior, the data have not been made publicly available. The data are available from the corresponding author on request.

\section{Declarations}

\section{Ethics approval and consent to participate}

The institutional review board at the Nigerian Federal Capital Territory Health Research Ethics Committee and the Institutional Review Board at the University of Maryland Baltimore reviewed and approved the research protocol. All participants provided written informed consent prior to enrollment and all data were de-identified.

\section{Consent for publication}

Not applicable.

\section{Competing interests}

OO declares no relevant conflicts of interest. All other authors declare no relevant conflicts of interest.

\section{Author details}

'Johns Hopkins Bloomberg School of Public Health, 615 North Wolfe Street, Suite 3507, Baltimore, MD 21205, USA. ${ }^{2}$ U.S. Military HIV Research Program, Walter Reed Army Institute of Research, Silver Spring, MD, USA. ${ }^{3}$ Henry M. Jackson Foundation for the Advancement of Military Medicine, Bethesda, MD, USA. Institute of Human Virology, University of Maryland School of Medicine, Baltimore, MD, USA. ${ }^{5}$ School of Public Health, University of Maryland, College Park, MD, USA. ${ }^{6}$ HJF Medical Research International, Abuja, Nigeria. ${ }^{7}$ U.S. Army Medical Research Directorate - Africa, Nairobi, Kenya. ${ }^{8}$ Maryland Global Initiatives Corporation (MGIC), Abuja, Nigeria.

Received: 10 July 2020 Accepted: 11 June 2021

Published online: 30 June 2021

\section{References}

1. Baral SD, Grosso A, Holland C, Papworth E. The epidemiology of HIV among men who have sex with men in countries with generalized HIV epidemics. Curr Opin HIV AIDS. 2014;9(2):156-67 https://doi.org/10.1097/COH. 0000000000000037.

2. Baral SD, Ketende S, Schwartz S, Orazulike I, Ugoh K, Peel SA, et al. Evaluating respondent-driven sampling as an implementation tool for universal coverage of antiretroviral studies among men who have sex with men living with HIV. J Acquir Immune Defic Syndr (1999). 2015;68(Suppl 2(0 2)):S107-13 https://doi.org/10.1097/QAl.0000000000000438.

3. Beyrer C, Baral SD, van Griensven F, Goodreau SM, Chariyalertsak S, Wirtz AL, et al. Global epidemiology of HIV infection in men who have sex with men. Lancet. 2012;380(9839):367-77 https://doi.org/10.1016/S0140-6736(12)608216.

4. Bingham TA, Harawa NT, Johnson DF, Secura GM, Mackellar DA, Valleroy LA, et al. The effect of partner characteristics on HIV infection among African American men who have sex with men in the young Men's survey, Los Angeles, 1999-2000. AIDS Educ Prev. 2003;15). Retrieved from https:// guilfordjournals.com/doi/pdf/10.1521/aeap.15.1.5.39.23613(1_supplement): 39-52.

5. Birkett M, Kuhns LM, Latkin C, Muth S, Mustanski B. The sexual networks of racially diverse young men who have sex with men. Arch Sex Behav. 2015; 44(7):1787-97 https://doi.org/10.1007/s10508-015-0485-5.

6. Cai Y, Wang Z, Lau JTF, Li J, Ma T, Liu Y. Prevalence and associated factors of condomless receptive anal intercourse with male clients among transgender women sex workers in Shenyang, China. J Int AIDS Soc. 2016; 19(3 Suppl 2) https://doi.org/10.7448/IAS.19.3.20800.

7. Crepaz N, Marks G, Mansergh G, Murphy S, Miller LC, Appleby PR. Agerelated risk for HIV infection in men who have sex with men: examination of behavioral, relationship, and serostatus variables. AIDS Educ Prev. 2000; 12(5):405-15 Retrieved from http://www.ncbi.nlm.nih.gov/pubmed/11063 060 .

8. Crowell TA, Baral SD, Schwartz S, Nowak RG, Kokogho A, Adebajo S, et al. Time to change the paradigm: limited condom and lubricant use among Nigerian men who have sex with men and transgender women despite availability and counseling. Ann Epidemiol. 2019;31:11-19.e3 https://doi. org/10.1016/j.annepidem.2018.12.004.

9. Crowell TA, Keshinro B, Baral SD, Schwartz SR, Stahlman S, Nowak RG, et al. Stigma, access to healthcare, and HIV risks among men who sell sex to men in Nigeria. J Int AIDS Soc. 2017;20(1):21489 https://doi.org/10.7448/IAS.20. 01.21489 .

10. Cuervo M, Whyte J. The effect of relationship characteristics on HIV risk behaviors and prevention strategies in young gay and bisexual men. J Assoc Nurses AIDS Care. 2015;26(4):399-410 https://doi.org/10.1016/j.jana.2 015.03.003.

11. Davidovich U, De Wit JBF, Stroebe W. Assessing sexual risk behaviour of young gay men in primary relationships: the incorporation of negotiated safety and negotiated safety compliance. AIDS. 2000;14(6):701-6 https://doi. org/10.1097/00002030-200004140-00009.

12. Davidovich U, De Wit JBF, Stroebe W. Behavioral and cognitive barriers to safer sex between men in steady relationships: implications for prevention strategies. AIDS Educ Prev. 2004;16(4):304-14 https://doi.org/10.1521/aeap.1 6.4.304.40398

13. Dosekun O, Fox J. An overview of the relative risks of different sexual behaviours on HIV transmission. Curr Opin HIV AIDS. 2010;5(4):291-7 https:// doi.org/10.1097/COH.0b013e32833a88a3.

14. Goldenberg T, Clarke D, Stephenson R. "Working together to reach a goal": MSM's perceptions of dyadic HIV care for same-sex male couples. J Acquired Immune Defic Syndr (1999). 2013;64(Suppl 1(0 1)):S52-61 https:// doi.org/10.1097/QAl.0b013e3182a9014a.

15. Greene GJ, Andrews R, Kuper L, Mustanski B. Intimacy, monogamy, and condom problems drive unprotected sex among young men in serious relationships with other men: a mixed methods dyadic study. Arch Sex Behav. 2014;43(1):73-87 https://doi.org/10.1007/s10508-013-0210-1.

16. Hart T, Peterson JL. Predictors of risky sexual behavior among young African American men who have sex with men. Am J Public Health. 2004;94(7): 1122-3 https://doi.org/10.2105/ajph.94.7.1122.

17. Hensel DJ, Rosenberger JG, Novak DS, Reece M. Sexual event-level characteristics of condom use during anal intercourse among HIV-negative men who have sex with men. Sex Transm Dis. 2012;39(7):550-5 https://doi. org/10.1097/olq.0b013e31824f1da4.

18. Hoff CC, Chakravarty D, Beougher SC, Neilands TB, Darbes LA. Relationship characteristics associated with sexual risk behavior among MSM in committed relationships. AIDS Patient Care STDs. 2012;26(12):738-45 https:// doi.org/10.1089/apc.2012.0198.

19. Hurt CB, Matthews DD, Calabria MS, Green KA, Adimora AA, Golin CE, et al. Sex with older partners is associated with primary HIV infection among men who have sex with men in North Carolina. J Acquired Immune Defic Syndr (1999). 2010;54(2):185-90 https://doi.org/10.1097/QAl.0b013e3181c99114.

20. Kelly JA, St. Lawrence JS, Amirkhanian YA, DiFranceisco WJ, Anderson-Lamb M, Garcia LI, et al. Levels and predictors of HIV risk behavior among black men who have sex with men. AIDS Educ Prev. 2013;25(1):49-61 https://doi. org/10.1521/aeap.2013.25.1.49.

21. Keshinro B, Crowell TA, Nowak RG, Adebajo S, Peel S, Gaydos CA, et al. High prevalence of HIV, chlamydia and gonorrhoea among men who have sex with men and transgender women attending trusted community centres in Abuja and Lagos, Nigeria. J Int AIDS Soc. 2016;19(1):21270 https://doi.org/10. 7448/IAS.19.1.21270. 
22. Kroenke K, Spitzer RL, Williams JB. The PHQ-9: validity of a brief depression severity measure. J Gen Intern Med. 2001;16(9):606-13 https://doi.org/10.104 6/J.1525-1497.2001.016009606.X.

23. Li D, Li C, Wang Z, Lau JTF. Prevalence and associated factors of unprotected anal intercourse with regular male sex partners among HIV negative men who have sex with men in China: a cross-sectional survey. PLoS One. 2015;10(3) https://doi.org/10.1371/journal.pone.0119977: e0119977.

24. Liu H. Egocentric network and condom use among mid-age female sex Workers in China: a multilevel modeling analysis. AIDS Patient Care STDs. 2016;30(4):155-65 https://doi.org/10.1089/apc.2015.0349.

25. McCree DH, Oster AM, Jeffries WL, Denson DJ, Lima AC, Whitman H, et al. HIV acquisition and transmission among men who have sex with men and women: what we know and how to prevent it. Prev Med. 2017;100:132-4 https://doi.org/10.1016/j.ypmed.2017.04.024

26. Mustanski B, Newcomb ME, Clerkin EM. Relationship characteristics and sexual risk-taking in young men who have sex with men. Health Psychol. 2011;30(5):597-605 https://doi.org/10.1037/a0023858.

27. Newcomb ME, Ryan DT, Garofalo R, Mustanski B. The effects of sexual partnership and relationship characteristics on three sexual risk variables in young men who have sex with men. Arch Sex Behav. 2014;43(1):61-72 https://doi.org/10.1007/s10508-013-0207-9.

28. Obi SN, Ifebunandu NA. Consequences of HIV testing without consent. Int J STD AIDS. 2006;17(2):93-6 https://doi.org/10.1258/095646206775455649.

29. Patel P, Borkowf CB, Brooks JT, Lasry A, Lansky A, Mermin J. Estimating peract HIV transmission risk: a systematic review. AIDS (London, England). 2014; 28(10):1509-19 https://doi.org/10.1097/QAD.0000000000000298.

30. Peterson JL, Rothenberg R, Kraft JM, Beeker C, Trotter R. Perceived condom norms and HIV risks among social and sexual networks of young African American men who have sex with men. Health Educ Res. 2009;24(1):119-27 https://doi.org/10.1093/her/cyn003.

31. Poppen PJ, Reisen CA, Zea MC, Bianchi FT, Echeverry JJ. Serostatus disclosure, seroconcordance, partner relationship, and unprotected anal intercourse among HIV-positive Latino men who have sex with men. AIDS Educ Prev. 2005;17(3):227-37 https://doi.org/10.1521/aeap.17.4.227.66530.

32. Poteat $T$, Ackerman B, Diouf $D$, Ceesay N, Mothopeng $T$, Odette $K Z$, et al. HIV prevalence and behavioral and psychosocial factors among transgender women and cisgender men who have sex with men in 8 African countries: a cross-sectional analysis. PLoS Med. 2017;14(11) https://doi.org/10.1371/ journal.pmed.1002422:e1002422.

33. Ramadhani HO, Ndembi N, Nowak RG, Ononaku U, Gwamna J, Orazulike I, et al. Individual and network factors associated with HIV care continuum outcomes among Nigerian MSM accessing health care services. J Acquired Immune Defic Syndr. 2018;79(1):e7-e16 https://doi.org/10.1097/QAl. 0000000000001756

34. Ramakrishnan L, Ramanathan S, Chakrapani V, Goswami P, Deshpande S, Yadav D, et al. Comparison of sexual risk, HIV/STI prevalence and intervention exposure among men who have sex with men and women (MSMW) and men who have sex with men only (MSMO) in India: implications for HIV prevention. AIDS Behav. 2015;19(12):2255-69 https://doi. org/10.1007/s10461-015-1058-2.

35. Rodriguez-Hart C, Liu H, Nowak RG, Orazulike I, Zorowitz S, Crowell TA, et al. Serosorting and sexual risk for HIV infection at the ego-Alter dyadic level: an egocentric sexual network study among MSM in Nigeria. AIDS Behav. 2016; 20(11):2762-71 https://doi.org/10.1007/s10461-016-1311-3.

36. Schneider JA, Cornwell B, Ostrow D, Michaels S, Schumm P, Laumann EO, et al. Network mixing and network influences most linked to HIV infection and risk behavior in the HIV epidemic among black men who have sex with men. Am J Public Health. 2013;103(1) https://doi.org/10.2105/AJPH.2 012.301003:e28-36.

37. Schwartz SR, Nowak RG, Orazulike I, Keshinro B, Ake J, Kennedy S, et al. The immediate eff ect of the same-sex marriage prohibition act on stigma discrimination, and engagement on HIV prevention and treatment services in men who have sex with men in Nigeria: analysis of prospective data from the TRUST cohort. Lancet. 2015;2(7):e299-306 https://doi.org/10.1016/ S2352-3018(15)00078-8. HIV.

38. Siegler AJ, de Voux A, Phaswana-Mafuya N, Bekker L-G, Sullivan PS, Baral SD, et al. Elements of condom-use decision making among south African men who have sex with men. J Int Assoc Providers AIDS Care. 2014;13(5):414-23 https://doi.org/10.1177/2325957414535979.
39. Strömdahl S, Onigbanjo Williams A, Eziefule B, Emmanuel G, Iwuagwu S, Anene $\mathrm{O}$, et al. Associations of consistent condom use among men who have sex with men in Abuja, Nigeria. AIDS Res Hum Retrovir. 2012;28(12): 1756-62 https://doi.org/10.1089/AID.2012.0070.

40. Sullivan PS, Salazar L, Buchbinder S, Sanchez TH. Estimating the proportion of HIV transmissions from main sex partners among men who have sex with men in five US cities. AIDS. 2009;23(9):1153-62 https://doi.org/10.1097/ QAD.0b013e32832baa34

41. Swartzendruber A, Zenilman JM, Niccolai LM, Kershaw TS, Brown JL, Diclemente RJ, et al. It takes 2: partner attributes associated with sexually transmitted infections among adolescents. Sex Transm Dis. 2013:40(5):372-8 https://doi.org/10.1097/OLQ.0b013e318283d2c9.

42. UNAIDS. (2014). THE GAP REPORT. Retrieved from http://www.unaids.org/ sites/default/files/media_asset/UNAIDS_Gap_report_en.pdf

43. Vu L, Adebajo S, Tun W, Sheehy M, Karlyn A, Njab J, et al. High HIV prevalence among men who have sex with men in Nigeria. J Acquired Immune Defic Syndr. 2013;63(2):221-7 https://doi.org/10.1097/QAl.0b013e31 $828 a 3 e 60$.

44. Vu L, Andrinopoulos K, Tun W, Adebajo S. High levels of unprotected anal intercourse and never testing for HIV among men who have sex with men in Nigeria: evidence from a cross-sectional survey for the need for innovative approaches to HIV prevention. Sex Transm Infect https://doi. org/10.1136/sextrans-2013-051065. 2013;89(8):659-65.

45. Wang H, Chang R, Shen Q, Tsamlag L, Zhang S, Shi Y, et al. Informationmotivation-behavioral skills model of consistent condom use among transgender women in Shenyang, China. BMC Public Health. 2020;20(1) https://doi.org/10.1186/s12889-020-08494-y:394.

46. Washington DC, Grootaert C, Narayan D, Jones VN, Woolcock M. The World Bank measuring social capital an integrated questionnaire; 2004.

47. Wei C, Raymond HF, Guadamuz TE, Stall R, Colfax GN, Snowden JM, et al. Racial/ethnic differences in seroadaptive and serodisclosure behaviors among men who have sex with men. AIDS Behav. 2011;15(1):22-9 https:// doi.org/10.1007/s10461-010-9683-2.

48. Yang J, Xu H, Li S, Cheng W, Gu Y, Xu P, et al. The characteristics of mixing patterns of sexual dyads and factors correlated with condomless anal intercourse among men who have sex with men in Guangzhou, China. BMC Public Health. 2019;19(1) https://doi.org/10.1186/s12889-019-7082-9: 722.

\section{Publisher's Note}

Springer Nature remains neutral with regard to jurisdictional claims in published maps and institutional affiliations.
Ready to submit your research? Choose BMC and benefit from:

- fast, convenient online submission

- thorough peer review by experienced researchers in your field

- rapid publication on acceptance

- support for research data, including large and complex data types

- gold Open Access which fosters wider collaboration and increased citations

- maximum visibility for your research: over $100 \mathrm{M}$ website views per year

At BMC, research is always in progress.

Learn more biomedcentral.com/submission 